\title{
Development of room temperature crossbar- $H$-mode cavities for proton and ion acceleration in the low to medium beta range
}

\author{
G. Clemente, ${ }^{1, *}$ U. Ratzinger, ${ }^{2}$ H. Podlech, ${ }^{2}$ L. Groening, ${ }^{1}$ R. Brodhage, ${ }^{2}$ and W. Barth ${ }^{1}$ \\ ${ }^{1}$ GSI Helmholtzzentrum für Schwerionenforschung GmbH, Darmstadt, Plankstraße 1, 64291 Darmstadt, Germany \\ ${ }^{2}$ Institute for Applied Physics (IAP), University of Frankfurt, 60438, Frankfurt, Germany
}

(Received 1 February 2011; published 10 November 2011)

\begin{abstract}
The crossbar $H$-mode $(\mathrm{CH})$ cavity is an accelerating structure operated in the $H_{21(0)}$ mode. The robustness of the crossbar geometry allows one to realize room temperature as well as superconducting linac cavities. The shunt impedance characteristics of this structure are attractive to develop proton and heavy ion linacs in the low and medium beta range. A first room temperature eight-cell prototype has proven the feasibility of the crossbar design in terms of mechanical construction, copper plating, and cooling. An innovative rf coupling concept has been developed where two $\mathrm{CH}$ cavities are connected by a two gap $E_{010}$-mode resonator which, at the same time, provides transverse focusing by a quadrupole triplet. The concept has been applied in the design of the new FAIR proton linac and a scaled model of the second cavity of this injector has been built and tested too. The full scale prototype is now under construction at the University of Frankfurt. In this paper, the room temperature $\mathrm{CH}$ cavity development as well as the general layout of the FAIR proton injector $(70 \mathrm{MeV}, 325 \mathrm{MHz}, 70 \mathrm{~mA})$ is presented and discussed.
\end{abstract}

DOI: 10.1103/PhysRevSTAB.14.110101

PACS numbers: 41.20.- q, 41.75.Ak, 41.85.Ew

\section{INTRODUCTION}

During the past 15 years an increasing activity in linac development came up due to the capability of this kind of accelerator to deliver high intensity, high duty factor, proton and ion beams. The main trigger for this new quest was the demand from nuclear, material, and biological sciences for neutron beams with energy and time spectra different from that available by power plants. The successful testing of the LEDA machine [1-3] at Los Alamos demonstrated the possibility to accelerate a $100 \mathrm{~mA}$ proton beam at a frequency as high as $350 \mathrm{MHz}$. That result opened new perspectives in ion and proton acceleration.

The R\&D activities on linear accelerators have found application in the $1 \mathrm{MW}$ beam facility SNS [4] and in many other linac designs, such as ESS [5], FRIB [6], FRANZ [7], EUROTRANS [8], and IFMIF [9]. In particular, related to those last two projects, there has been an increasing demand to develop superconducting cavities for the low and medium beta range for $\mathrm{cw}$ application.

On the other hand, the interest towards pulsed operation has been active in designing high intensity injectors for synchrotrons, for instance at JPARC [10] and at CERN (LINAC4 [11]). Both projects base the front end on a $3 \mathrm{MeV}$ radio frequency quadrupole (RFQ) cavity followed by a three tank Alvarez drift tube linac (DTL)

\footnotetext{
*g.clemente@gsi.de
}

Published by the American Physical Society under the terms of the Creative Commons Attribution 3.0 License. Further distribution of this work must maintain attribution to the author(s) and the published article's title, journal citation, and DOI. up to the energy of $50 \mathrm{MeV}$. The Alvarez DTL with a transversal focusing focusing-drift-defocusing-drift lattice still represents the standard solution for proton acceleration, but the $E_{010}$ mode and the necessity to host a magnetic quadrupole in every drift tube considerably increases the efforts in mechanics and rf tuning. The integration of magnetic quadrupoles results in strict drift tube alignment tolerances, in the order of less than $0.1 \mathrm{~mm}[12]$.

The most useful alternative to the Alvarez linac in the low beta range is the interdigital $H$-mode linac (IH-DTL) which has been established as a standard cavity for ion acceleration in the frequency range from 30 to $220 \mathrm{MHz}$. The IH-DTL with quadrupole triplet focusing has different advantages, the higher shunt impedance and thus the low operation costs, a reduced number of quadrupoles, robustness against beam losses, a small overall size, and the simplified mechanical design which results in reduced fabrication costs $[13,14]$.

In order to extend the use of $H$-mode cavities to the higher resonance frequencies required for a hundred $\mathrm{MeV}$ booster, the crossbar $H$-mode (CH)-DTL has been developed for several years at GSI and at Frankfurt University [15]. The results in terms of rf efficiency and technical feasibility have been very promising. Therefore, GSI intends to base the upgrade of its linear accelerators on this novel cavity. In particular, the $\mathrm{CH}$ structure will be used for the new FAIR proton injector and it is a candidate for the future upgrade of the existing UNILAC [16]. Additionally, a superconducting, energy variable cw linac [17] was designed to improve the GSI experimental capabilities in nuclear chemistry and in super heavy elements (SHE) 


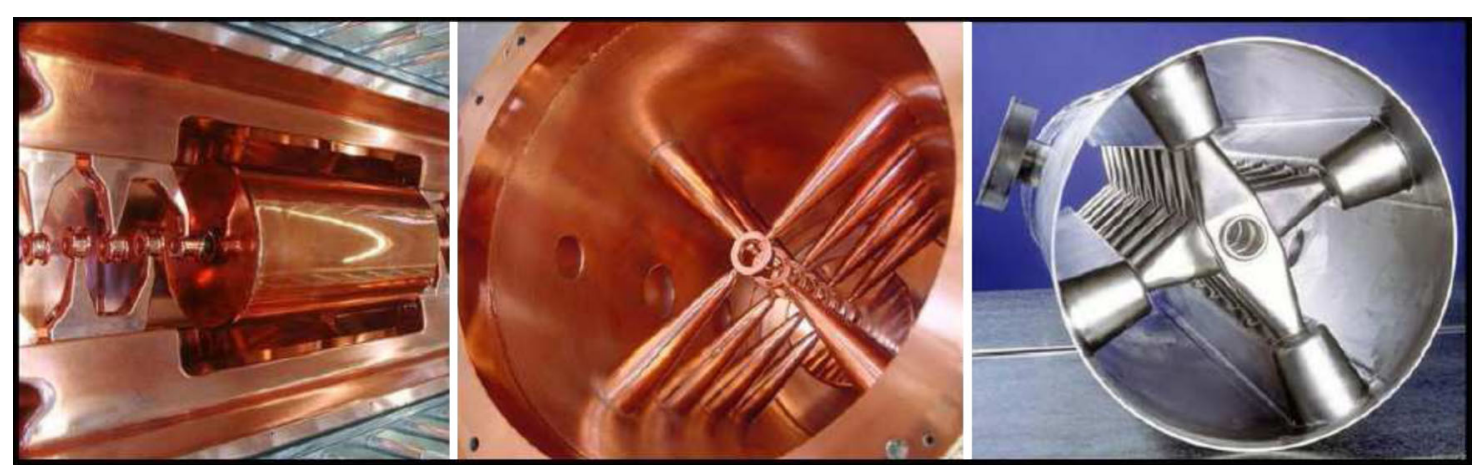

FIG. 1. The family of $H$-mode DTL's. From left to right: the IH-DTL, the room temperature, and the superconducting version of the CH-DTL.

synthesis. The use of room temperature $\mathrm{CH}$ cavities is investigated not only at GSI but at Fermilab [18] and at LANL [19] too.

Figure 1 shows the $H$-mode family of DTL.

\section{THE ROOM TEMPERATURE CH-DTL}

\section{A. Overview}

As cavities operated in the $E$ mode provide by definition a dominant component of the electric field along the beam axis, most linear accelerators are related to that modes' family. In Alvarez-type DTL the electric field has the same phase in every cell and the whole drift tubes array operates in a 0 mode. A FODO or focusing-drift-focusing-driftdefocusing-drift-defocusing-drift focusing scheme can be provided by the insertion of a quadrupole magnet inside every drift tube. That represents a serious constraint at low beam energies as the drift tubes are rather short and less space is available for the magnets. At the same time, the large diameter of the drift tubes contributes to decrease the rf efficiency and limits the achievable effective field gradient.

Additionally, together with the constant longitudinal field $E_{010}$ mode, along the structure a linearly increasing field mode exists (see, e.g., [20]). This linear mode is in general exited by local perturbations resulting in a distortion of the axial electric field. Inside a cavity, small fabrication errors, temperature gradients, or mechanical deformations could easily result in a large change of the gap field distribution. This problem becomes particularly serious as the length of the DTL increases because the frequency separation between modes gets lower. This is mostly the case as longer tanks are generally built for an effective and economic use of the power supplies. For that reason, DTL's are equipped with movable tuners and fixed postcouplers which are mounted against every or every third drift tube. The use of postcouplers imposes an important constraint as, in order to ensure an effective stabilization effect, they require a spacing between the cavity wall and the drift tube outer radius lower than $1.01 \cdot \lambda / 4$ (see, e.g., $[21,22]$ ). This limits the possibility to reduce the outer radius of the drift tubes and thus to increase the shunt impedance.
There is another observation concerning the drift tube diameter in DTL's, which is particularly important in the case of the separated function DTL (SDTL). In fact, those cavities are typically very short and no field stabilization is then required. This allows one to reduce the drift tube diameter and thus to increase the shunt impedance. On the other hand, as the bore aperture should be quite large due to the long focusing period, a reduced ratio between the beam aperture and the drift tube diameter strongly limits the possibility to shape the face angle and the nose radius of the drift tube. Those are in fact the main parameters to control the surface peak field. For example, at JPARC the ratio between the drift tube diameter $(92 \mathrm{~mm})$ and the beam aperture $(36 \mathrm{~mm})$ is 2.55 . A possible solution to this problem is to reduce the field gradient $E_{0}$ with the main consequence of an increase of the number of cavities and rf amplifiers.

Moreover, the long focusing period imposes to reduce the number of gaps. This results also in a reduction of the shunt impedance due to the effect of the end walls (Fig. 2). If this effect is nevertheless limited for high betas, it turns

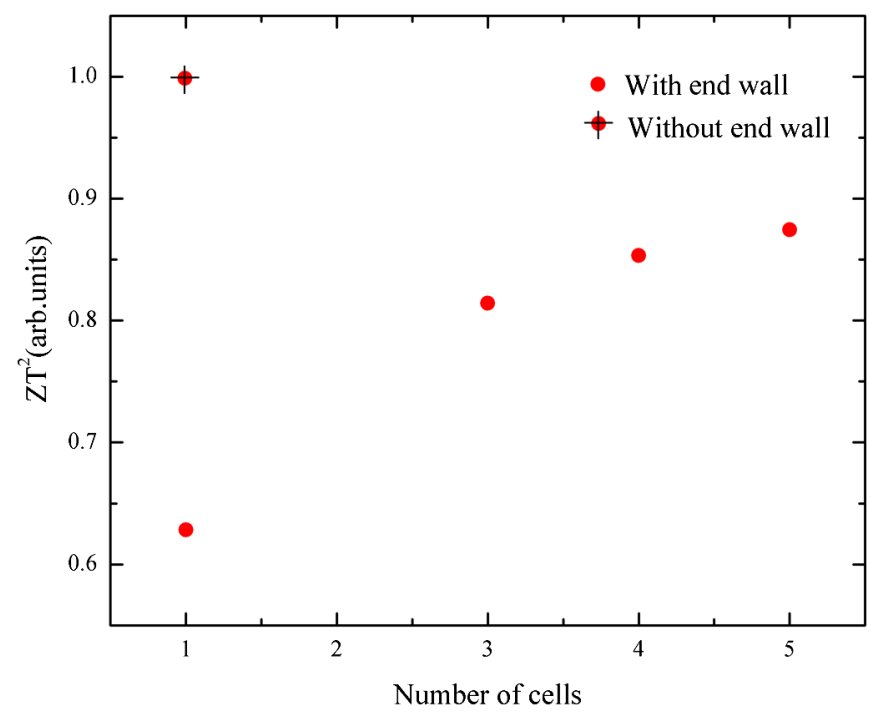

FIG. 2. The dependence of the shunt impedance from the number of cells for an SDTL for $\beta=0.3$ at $325 \mathrm{MHz}$. 
in a serious constraint at lower velocities. Additionally, as the focusing period becomes very long for small betas, those cavities are not suitable for acceleration of low energies and increasing current beams.

Nevertheless, Alvarez DTL's are, at present, the most valid proven solution concerning proton acceleration. The intense R\&D activities at SNS, JPARC, and CERN led to many improvements which fully justify the use of those cavities for the acceleration of high intensity proton beams.

A valid alternative to overcome the DTL problems related to the high fabrication costs and field sensitivity is represented by the cavities belonging to the $H$-mode family. In particular, the IH-DTL has proven to be a full reliable and performant solution for heavy ion acceleration while, as explained later on, the recent development of the CH-DTL could lead to an alternative solution concerning proton acceleration in the low to medium beta profile.

$H$-mode cavities are characterized by the direction of the magnetic field which is oriented parallel and antiparallel to the beam axis along the cavity sectors. Field lines with opposite orientation are connected at the cavity ends providing the required closed field loops. IH-DTL's are excited in the $H_{11(0)}$ mode while the use of the $H_{21(0)}$ mode results in the typical crossbar geometry of the CH-DTL. In particular, the crossbar geometry ensures the mechanical stability required for superconducting operation [23].

In contrast to cavities operated in the $E_{010}$ mode, $H$ cavities profit a lot from a slim drift tube geometry with reduced outer radius as the contribution of the drift tube capacity to the total capacity is significant for $H$ modes. In this way the longitudinal electric fields are concentrated around the beam axis. The simple architecture of the drift tubes allows one to increase the resonance frequency at low beam energies with the overall effect of a considerable increase of the shunt impedance. As an illustrative example, at $325 \mathrm{MHz}$ the corresponding pillbox cavities for the $E_{010}$ and $H_{210}$ modes have an outer radius $R_{p b}$ of 352 and $448 \mathrm{~mm}$, respectively. As shown in Fig. 3 the insertion of the acceleration structure (cylindrical drift tubes with beam aperture of $20 \mathrm{~mm}$ and stems) leads to a massive reduction of the frequency for the $H_{210}$ mode while a reduction of less than $25 \%$ should be expected for a reasonable DTL geometry in the $300 \mathrm{MHz}$ region.

For instance, for the JPARC DTL [24] and for CERN LINAC4, the insertion of the drift tube structure leads to a reduction of the resonance frequency by $20 \%$ compared to the corresponding pillbox cavity. At lower frequencies this effect is much more reduced: for example, for the $108 \mathrm{MHz}$ UNILAC at GSI, the corresponding reduction is only $8 \%$.

Moreover, it has been demonstrated that a very high Kilpatrick factor $(\approx 3.5$ at $200 \mathrm{MHz})$ can be reached [25] if the beam pulse is sufficiently short $(\approx 1 \mathrm{~ms})$, resulting in a very high effective accelerator gradient (up to $10 \mathrm{MV} / \mathrm{m}$ ). The use of the KONUS beam dynamics [26] allows one to maximize the applied acceleration voltage

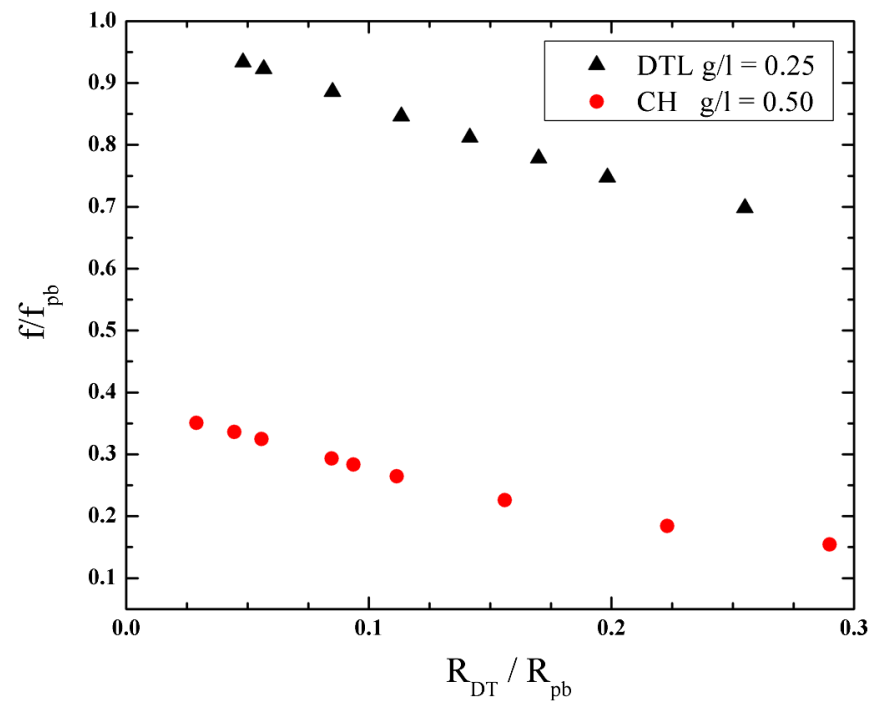

FIG. 3. The effect on the insertion of the accelerating structure on the resonance frequency of the corresponding pillbox cavity for an Alvarez DTL and for a CH-DTL.

between two focusing elements. As shown in Fig. 4, a KONUS period consists of: (1) a transverse focusing lens (solenoid or quadrupole triplet); (2) a short negative synchronous particle section for longitudinal matching; (3) a main acceleration section along a $0^{\circ}$ structure with asynchronous beam injection.

The heart of KONUS consists in the main acceleration section (3) defined by a $0^{\circ}$ synchronous particle: in this section the bunch center is injected with increased energy relatively to the $0^{\circ}$ synchronous particle. Because of this extra energy, the bunch center will encounter the next gap when the
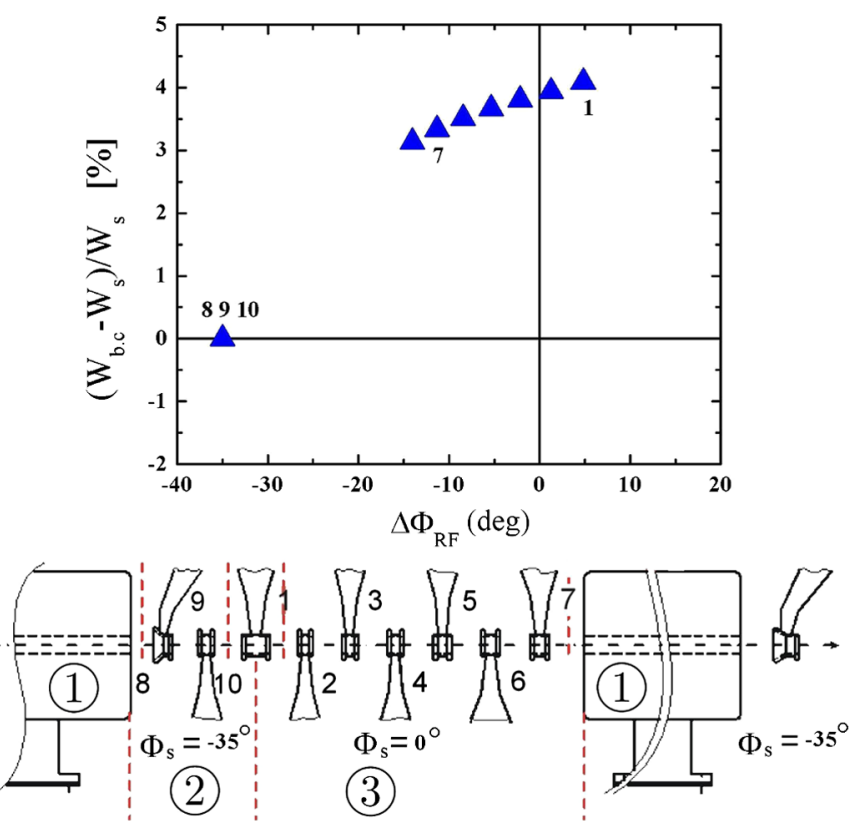

FIG. 4. The motion of the bunch center in the phase space in a typical KONUS period. 
field is not on the top and with a more negative phase, thus reducing the energy difference with respect to the synchronous particle. This corresponds to a counterclockwise motion in the longitudinal phase space. A longitudinal stable motion occurs as long as the bunch center is confined in the second quadrant since, only in this region of the phase space, earlier particles encounter a lower electric field with respect to the bunch center. Before the bunch is entering into the third quadrant, the drift tube structure is terminated and followed by a lens (1. This focusing element is generally a quadrupole triplet for room temperature operation while a superconducting solenoid is preferred for superconducting design.

The bunch lengthening along the drift needed for the magnetic lens is reduced by matching the beam with a short negative synchronous particle section (2) to the next main accelerating section (3.

The use of the KONUS beam dynamics is particularly useful only when applied to $H$-mode cavities because, as already explained in Fig. 3, in those cavities the contribution of the total capacitance coming from the accelerating structure is much higher than in standard DTL's. Even if also DTL's profits, at high frequency, from a thin drift tube geometry, the necessity to install the postcouplers limits the possibility to reduce the size of the drift tubes. This makes a standard beam dynamics scheme preferable. At high velocities, where a more relaxed focusing can be provided, the standard solution is to build long arrays of few gap cavities followed by quadrupole doublets. At JPARC SDTL cavities made of five gaps were realized to cover the energy range from 50 to $200 \mathrm{MeV}$. Nevertheless, as those cavities cannot be coupled, there is a need for a large number of power supplies or for power splitters from a single large klystron. In this latter case phase and amplitude control systems along the high power line must be carefully designed. This is also why alternative solutions, based on coupled cavities, such as PIMS or CCDTL's, have been developed at CERN.

The use of KONUS is then mainly suited for $\mathrm{H}$-mode cavities where it helps to minimize the electric capacitance. This allows the construction of lens-free multigap sections transversally matched by a quadrupole triplet. At energies above $30 \mathrm{MeV}$, a single large klystron can be efficiently matched to a single lens-free $\mathrm{CH}$ cavity.

Based on this scheme IH cavities with up to 56 gaps [27] and with three internal triplets have been realized while operation of the $20 \mathrm{~mA} \mathrm{U}^{4+}$ high current injector (HSI) at GSI has been demonstrating that high beam currents can be accelerated by this technique $[28,29]$.

The IH-DTL represents the state of the art for ion acceleration in terms of $\mathrm{rf}$ efficiency in the velocity range from $\beta \approx 0.01$ to 0.2 . For $\beta \leq 0.1$ it is competitive with superconducting structures concerning the plug power demands, at identical accelerating length, even at $\mathrm{cw}$ operation.

The only limitation concerning the use of the IH-DTL for a hundred $\mathrm{MeV}$ machine is given by the $\beta^{-1}$

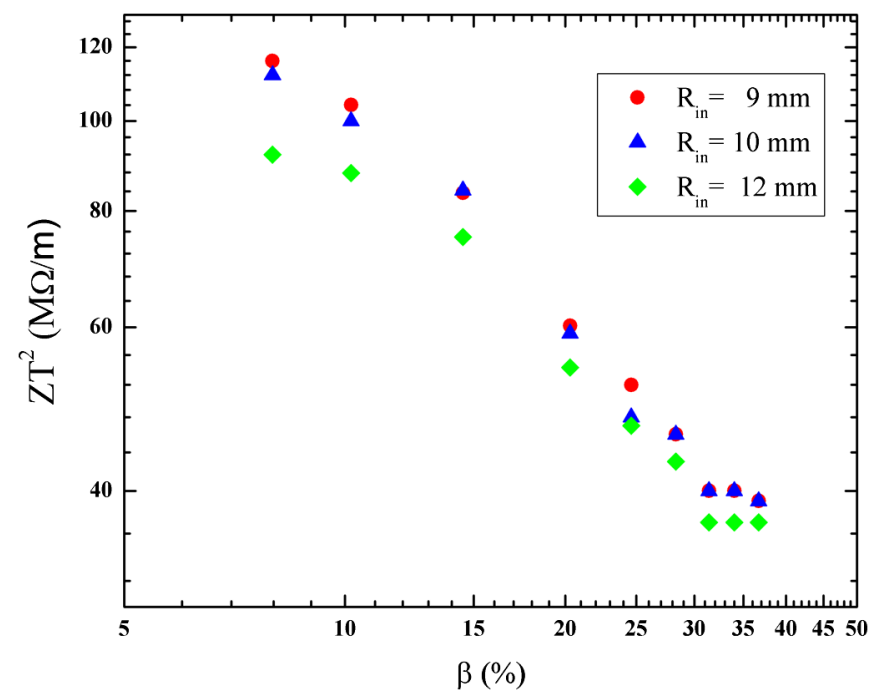

FIG. 5. Effective shunt impedance as a function of the velocity profile for drift tube apertures of 18,20 , and $24 \mathrm{~mm}$ at the resonance frequency of $325 \mathrm{MHz}$.

dependence [30] of the shunt impedance and by the small transverse dimensions at higher frequencies which allow only the construction of cavities with a reduced cell number. Because of that, it is natural to choose the $H_{21(0)}$ mode which provides simultaneously increased cavity diameters at a given frequency and shows also attractive mechanical features.

The crossbar geometry could allow the design of multicell structures even up to the $\mathrm{GHz}$ range [31]. At a frequency of $325 \mathrm{MHz}$, calculations have shown very high values of the effective shunt impedance in comparison with a traditional Alvarez DTL in the low to medium velocity profile. Figure 5 shows single cell calculations of the effective shunt impedance per unit length as a function of the velocity range for two different drift tube apertures. At each step the resonance frequency has been kept fixed by adapting the outer radius and the $\mathrm{g} / \mathrm{l}$ ratio was kept constant at 0.5 . The conductivity of the structure was set to $5.7 \times 10^{7} \mathrm{~Sv}$, i.e., the nominal value for copper at room temperature.

Figure 6 compares the calculated effective shunt impedance of the six SNS DTL cavities [32] with the JPARC (S) DTL cavities [33], the CERN LINAC4 DTL cavities [34], and the CH-DTL FAIR proton injector. A safety margin of $20 \%$ is included in the simulation concerning the DTL's and the CH-DTL's.

Those results open the perspective to adopt the $\mathrm{CH}$ structure for ion acceleration up to and even beyond $100 \mathrm{AMeV}$.

\section{B. Analytical field analysis}

$H$-mode accelerators are characterized by a high capacitive load compared to the corresponding empty cavity. The insertion of the drift tube structure leads to a drastic 


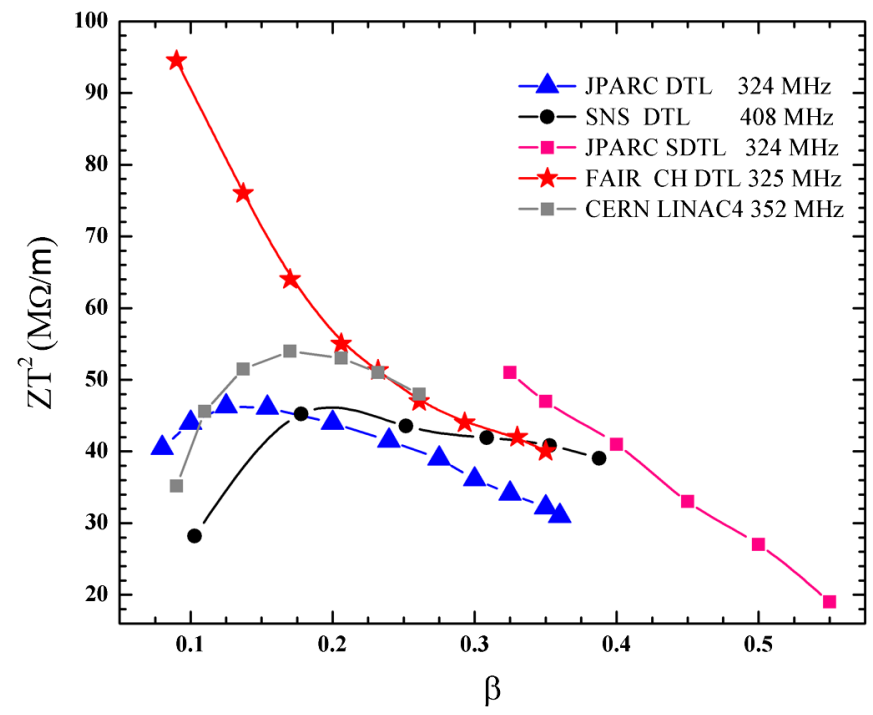

FIG. 6. A comparison of the effective shunt impedance of recent proposed and built DTL's.

modification of the electric field distribution which is concentrated mainly close to the beam axis. In empty cavities the electromagnetic fields can be derived from Bessel functions which is not possible in case of $\mathrm{CH}$ structures. Figure 7 shows the radial dependence of the magnetic field for an empty cylindrical cavity (CH-mode: $H_{211}$ ) and for two $\mathrm{CH}$ structures with geometrical betas of 0.07 and 0.25 . The significant change in the field distribution can be clearly seen. Additionally, the field distribution depends on the geometrical $\beta$ of the cavity.

First analytical descriptions of the electromagnetic field distribution inside a $\mathrm{CH}$ structure can be found in $[35,36]$. In this section that description is extended by taking into account the dependence of the electromagnetic field distribution from $\beta$. Analytical expressions for the electromagnetic fields are used to calculate the stored energy and, in a final step, the resonance frequency is derived. Because of the complexity of the problem, in the first step,

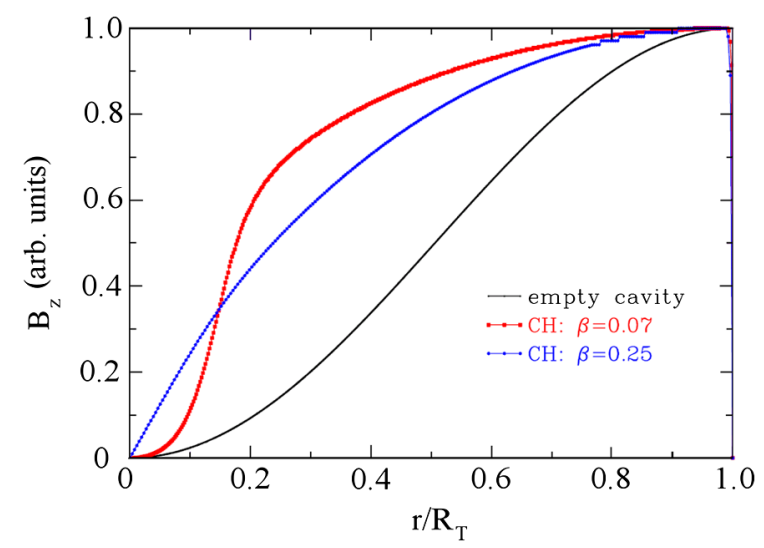

FIG. 7. Magnetic field $B_{z}$ for an empty cavity ( $H_{211}$ mode) and for two $\mathrm{CH}$ structures with geometrical betas of 0.07 and 0.25 .

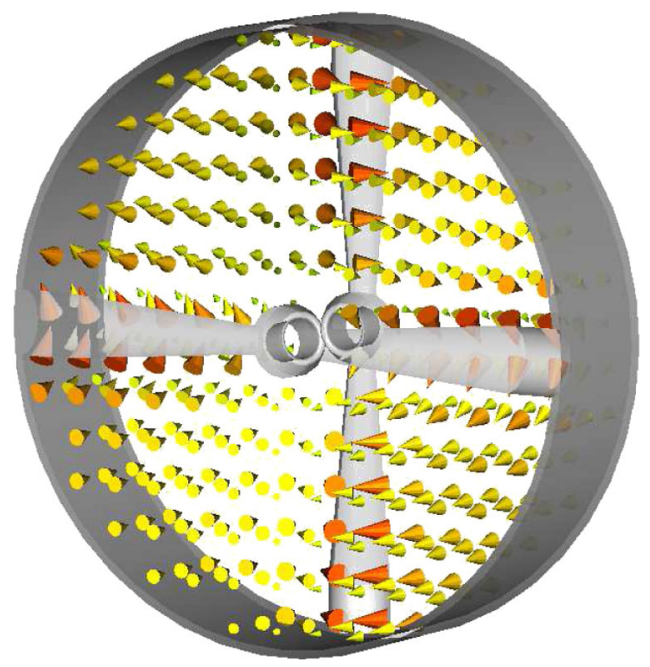

FIG. 8. Magnetic field in a $\beta=0.25 \mathrm{CH}$ structure.

electromagnetic simulations of $\mathrm{CH}$ cavities with different geometrical $\beta$ were necessary to evaluate the field distribution.

In all calculations the aperture radius $r_{i}$ is $12.5 \mathrm{~mm}$, the outer drift tube radius $r_{a} 20 \mathrm{~mm}$, and the $\mathrm{g} / \mathrm{L}$ ratio is 0.5 . The rf frequency was kept constant at $325 \mathrm{MHz}$ by adapting the tank radius $R_{T}$ for the different $\beta$. As an example, Fig. 8 shows the magnetic field distribution inside a $\mathrm{CH}$ cavity with $\beta=0.25$. The schematic model used for the calculations is then shown in Fig. 9.

In each quadrant the time varying magnetic flux creates the electric field components in the $\varphi$ direction. Although the magnetic field distribution is not homogeneous, as a starting point a homogeneous field $B_{z}=B_{0}$ was assumed,

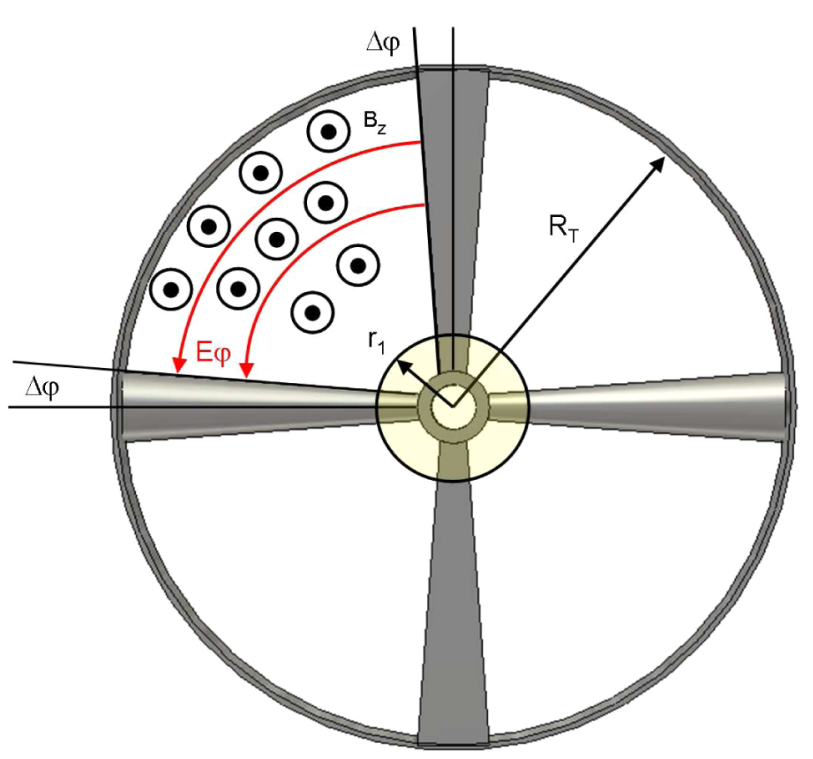

FIG. 9. Schematic view of a $\mathrm{CH}$ structure used for the analytical model. 
being $B_{0}$ the magnetic field at $r=R_{T}$. The drift tubes are charged as a consequence of the induction law, thus the potential $U_{i}$ can be expressed as

$$
\begin{gathered}
\left|U_{i}\right|=\dot{\Phi}=\iint B(t) d A \\
\left|U_{i}\right|=\int_{\Delta \varphi}^{\pi / 2-\Delta \varphi} \int_{r_{1}}^{R_{T}} B_{0} \mathrm{e}^{i \omega t} r d r d \varphi \\
\left|U_{i}\right|=\frac{\omega B_{0}}{2}\left(\frac{\pi}{2}-2 \Delta \varphi\right)\left(R_{T}^{2}-r_{1}^{2}\right) .
\end{gathered}
$$

It is assumed that the magnetic field can be neglected for $r \leq r_{1}$ (see Fig. 10). Using the path integral along the electric field lines

$$
U_{i}=\oint E_{\varphi} d s=\int_{\Delta \varphi}^{\pi / 2-\Delta \varphi} E_{\varphi}(r) r d \varphi
$$

it is possible to evaluate the electric field by comparing 3 with 4:

$$
E_{\varphi}(r)=\frac{\omega B_{0}}{2} \frac{R_{T}^{2}-r^{2}}{r}
$$

The maximum electric field $E_{0}$ exists at $r=r_{1}$ because of the maximum magnetic flux. Within $r_{1}$ the electric field is considered to be constant.

By plotting $B_{z}^{2} r$ versus $r$, one can determine the value of $r_{1}$ together with a correction factor $c_{1}$ which takes into account the real inhomogeneity of the magnetic field between $R_{T}$ and $r_{1}$. Figure 10 shows $B_{z}^{2} r$ versus $r$ for different $\beta$ obtained by rf simulations (thick curves). The area below these curves corresponds to the magnetic flux. In case of a homogeneous field distribution, a linear function is expected. Assuming a homogeneous field of magnitude $B_{0}$ will result in an overestimation of the induced voltage and the stored magnetic energy. The thin curves represent the tangents at the inflection points of the thick curves. The

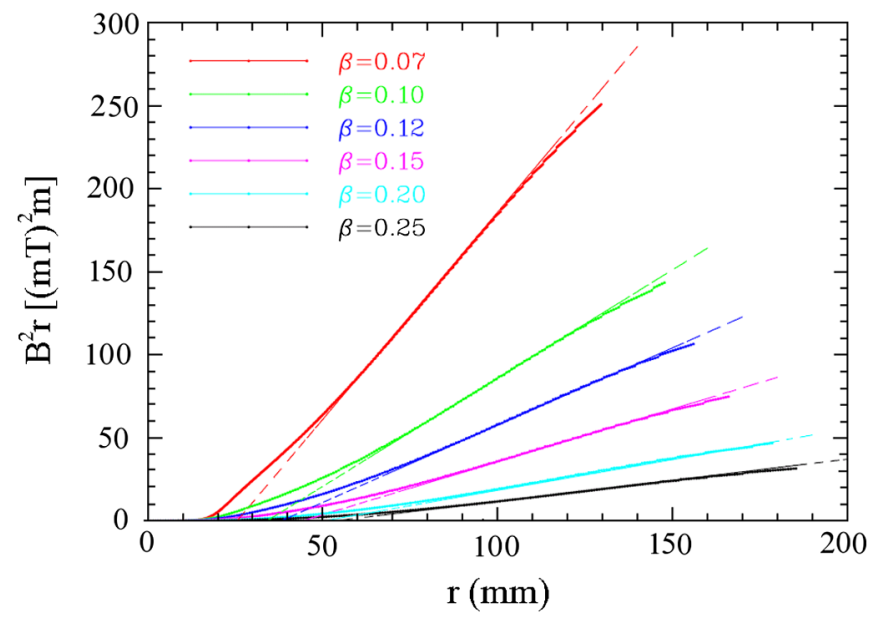

FIG. 10. $B_{z}^{2} r$ versus $r$ for different $\beta$. intersections between the tangents and the $x$ axis give the radius $r_{1}$ where the magnetic field can be neglected.

The magnetic field at the inflection points $\left(B_{i p}\right)$ reveals to be the correct choice in order to obtain the average magnetic field. The corresponding correction factor $c_{1}$ is then $B_{i p} / B_{0}$. The value of $c_{1}$ is $0.92 \pm 0.02$.

Now it is possible to calculate the stored magnetic energy within one accelerating cell with a length of $\beta \lambda / 2$. Two different volumes have to be considered, the four quadrants and the space between the stems. The magnetic energy $W_{m 1}$ in the quadrants is

$$
W_{m 1}=\frac{1}{2 \mu_{0}} \int_{V}|B|^{2} d V
$$

$$
\begin{aligned}
& W_{m 1}=4 \frac{1}{2 \mu_{0}} \int_{r_{1}}^{R_{T}} \int_{\Delta \varphi}^{\pi / 2-\Delta \varphi} \int_{0}^{\beta \lambda / 2}\left(c_{1} B_{0}\right)^{2} r d r d \varphi d z \\
& W_{m 1}=\frac{\left(c_{1} B_{0}\right)^{2}}{\mu_{0}}\left(\frac{\pi}{2}-2 \Delta \varphi\right)\left(R_{T}^{2}-r_{1}^{2}\right) \frac{\lambda \beta}{2} .
\end{aligned}
$$

In the space between the stems the magnetic field is enhanced in this region compared to $B_{0}$ because of the large currents on the stem surface. The enhancement should be higher for larger $\beta$ because more current per stem is required to charge the drift tubes. Simulations showed that the enhancement can be described via a linear function of $\beta$ as shown in Fig. 11.

Additionally, the field between two stems is in good approximation linear as shown in Fig. 12.

Taking all these considerations into account, the stored magnetic energy $W_{m 2}$ between the stems can be calculated as

$$
W_{m 2}=\frac{2}{\mu_{0}} \int_{r_{1}}^{R_{T}} \int_{-\Delta \varphi}^{\Delta \varphi} \int_{0}^{\beta \lambda / 2-r_{s} / 2} B^{2}(z) d r d \varphi d z
$$

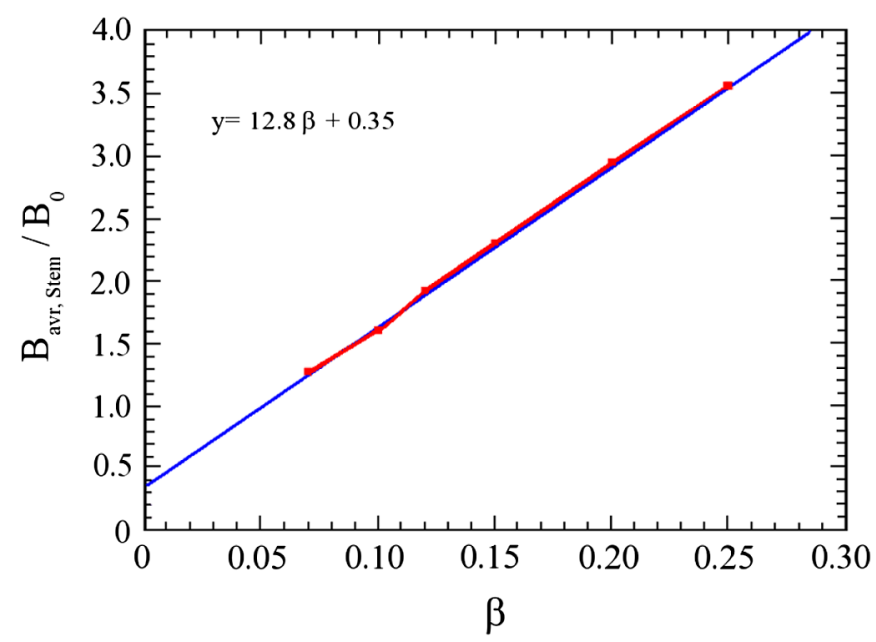

FIG. 11. Enhancement of the magnetic field in the vicinity of the stems as a function of the geometrical cavity $\beta$. The red dots are results of simulations and the blue curve is a linear fit. 


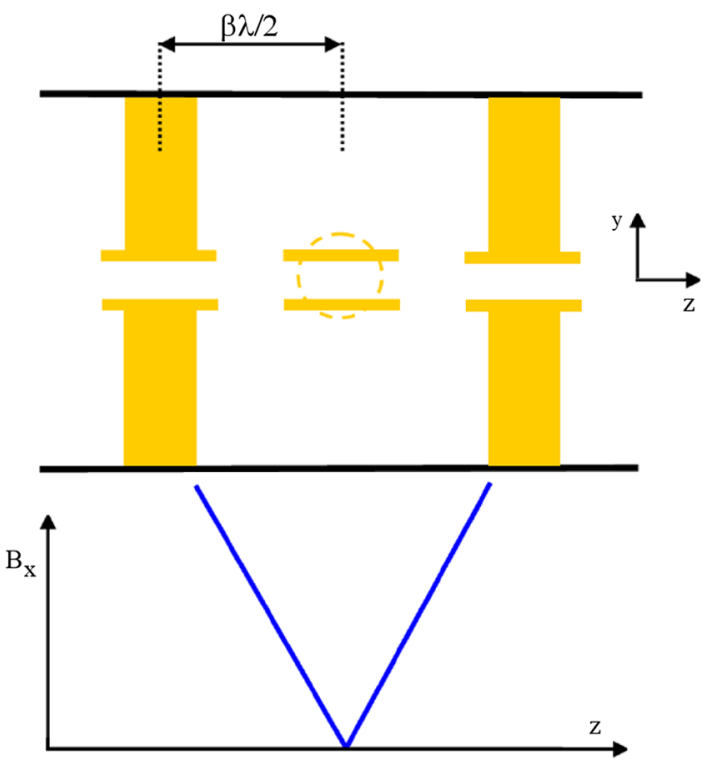

FIG. 12. Linear dependence of the magnetic field between the stems.

where $r_{s}$ is the stem base radius. $B(z)$ can be expressed by

$$
B(z)=B_{0}(12.8 \beta+0.35) \frac{z}{\beta \lambda / 2-r_{s} / 2} .
$$

Using $r_{s} \approx \Delta \varphi R_{T}$ the stored energy between the stems results in

$$
\begin{aligned}
W_{m 2}= & \frac{B_{0}^{2}}{\mu_{0}}\left(R_{T}^{2}-r_{1}^{2}\right)\left[\frac{2}{3}(12.8 \beta+0.35)^{2}\right. \\
& \left.\times\left(\frac{\beta \lambda}{2}-\Delta \varphi R_{T} / 2\right) \Delta \varphi+(\pi / 2-2 \Delta \varphi) \frac{\beta \lambda}{2}\right]
\end{aligned}
$$

The stored electric energy can be calculated using the field given in (5). One has to distinguish between the field in the quadrants and within the radius $r_{1}$. The stored energy $W_{e 1}$ in the four quadrants is given by

$W_{e 1}=\frac{\epsilon_{0}}{2} \int_{V} E_{\varphi}^{2}(r) d V$

$W_{e 1}=2 \epsilon_{0} \int_{r_{1}}^{R_{T}} \int_{\Delta \varphi}^{\pi / 2-\Delta \varphi} \int_{0}^{\beta \lambda / 2}\left(\frac{\omega B_{0}}{2} \frac{R_{T}^{2}-r^{2}}{r}\right)^{2} r d r d \varphi d z$

$W_{e 1}=\frac{\beta \lambda}{2} \epsilon_{0} \omega^{2} B_{0}^{2}\left(\frac{\pi}{2}-2 \Delta \varphi\right) R_{T}^{2}\left\{R_{T}^{2}\left[\ln \left(\frac{R_{T}}{r_{1}}\right)-\frac{3}{4}\right]+r_{1}^{2}\right\}$.

The stored energy $W_{e 2}$ of the electric field for $r \leq r_{1}$ can be calculated using

$$
E\left(r_{1}\right)=\frac{\omega B_{0}}{2} \frac{R_{T}^{2}-r_{1}^{2}}{r_{1}}
$$

which results in

$$
\begin{aligned}
& W_{e 2}=\frac{\epsilon_{0}}{2} \int_{0}^{r_{1}} \int_{0}^{2 \pi} \int_{0}^{\beta \lambda / 2} E\left(r_{1}\right)^{2} r d r d \varphi d z \\
& W_{e 2}=\frac{1}{8} \epsilon_{0} \pi \omega^{2} B_{0}^{2} \frac{\beta \lambda}{2}\left(R_{T}^{2}-r_{1}^{2}\right)^{2} .
\end{aligned}
$$

Assuming that there is no magnetic field within the drift tubes and in the aperture, the value of $W_{e 2}$ is lower by the factor of $r_{a}^{2} /\left(2 r_{1}^{2}\right)$. Figure 13 shows the stored energies $W_{m 1}, W_{m 2}, W_{e 1}, W_{e 2}$ and the total electric and magnetic energy as a function of $\beta$.

The rf frequency can be calculated by equalizing the total stored magnetic and electric field energy and solving for the frequency. Figure 14 shows the analytically calculated frequency as a function of $\beta$.

The agreement is excellent with a relative deviation of less than $2 \%$ except for very low $\beta$. In this case the neglect of the penetration of the electric field into the drift tubes

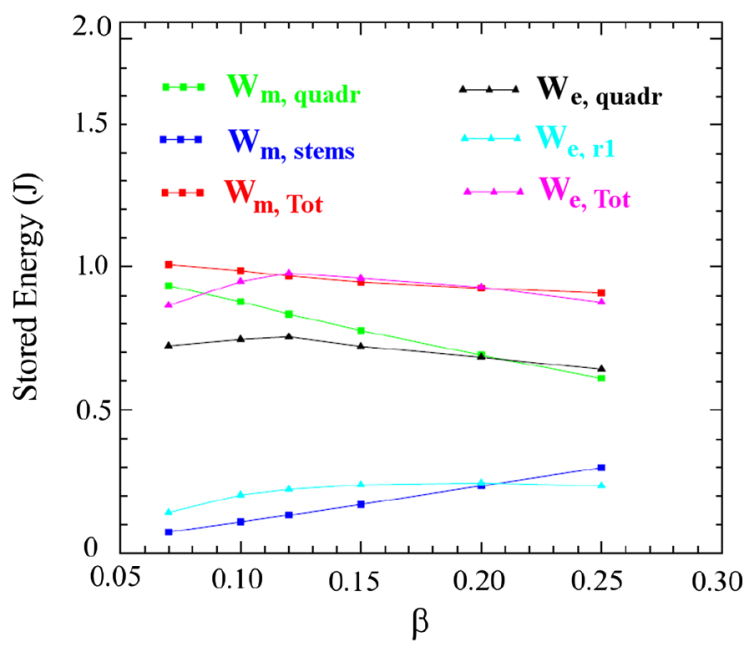

FIG. 13. Analytically calculated stored energies as a function of $\beta$.

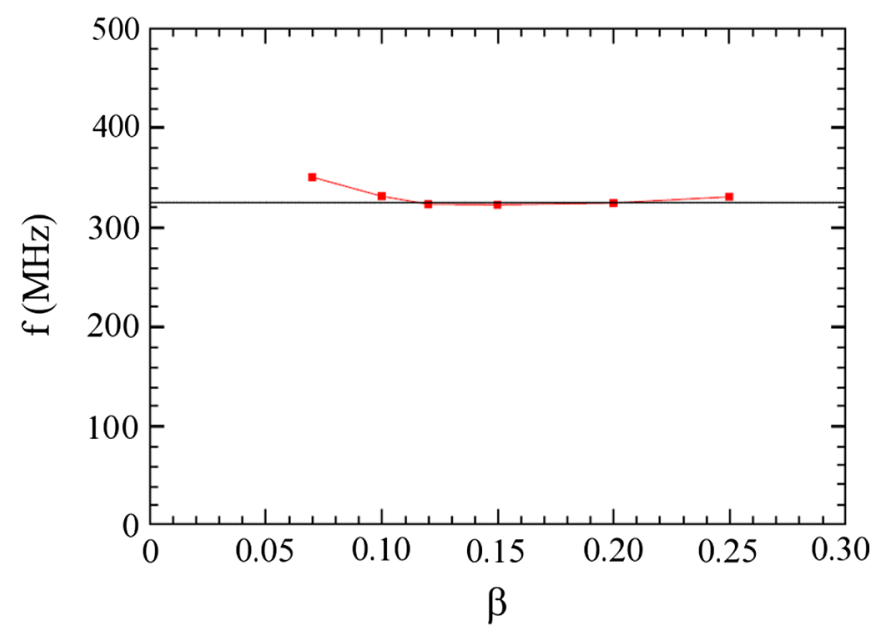

FIG. 14. Analytically calculated rf frequency as a function of $\beta$ in comparison to the simulated cavities tuned at $325 \mathrm{MHz}$. 
leads to a lower calculated stored energy and therefore to a higher frequency.

\section{CONSTRUCTION AND TEST MODEL}

In order to prove the technical feasibility of the crossbar geometry with respect to construction, cooling capabilities, and copper plating, a first model has been built at Frankfurt University [37].

The stainless steel cavity consists of eight cells with a constant period of $\beta \lambda / 2=45 \mathrm{~mm}$ for a total inner length of $600 \mathrm{~mm}$. The cavity wall diameter is $332 \mathrm{~mm}$ while the drift tubes have a constant aperture of $20 \mathrm{~mm}$. The copper plating operation of that complicated geometry was successfully performed at the GSI galvanic workshop.

\section{A. The stem}

In the $\mathrm{CH}$ structure currents flow from the outer cylinder to the drift tube to generate the axial electric field needed for particle acceleration. As a result, the stems stand the highest current density inside the cavity and, for that reason, the design must include a water channel. In order to minimize the rf losses due to nonperfect contacts, the stems are directly welded into the outer cylinder. This implies a robust design in order to stand the mechanical stress generated during the welding operations. On the other hand, close to the beam axis the stems have to be kept slim to reduce the capacitive load.

To take into account those constraints, a conical geometry has been chosen where the larger radius is located on the contact point with the outer cylinder. This solution reduces the capacitive load between neighbored stems and offers the required mechanical stability. Moreover, the resulting smooth geometry is also favorable for the galvanic copper plating.

\section{B. Field distribution}

The empty $H_{211}$ mode cavity shows a sinusoidal distribution of the longitudinal component $B_{z}$. When the stem structure is inserted into the empty cavity, the accelerating voltage $U_{n}$ of the $n$th gap can be calculated by the integration of the Faraday's law over a cavity quadrant $S$ :

$$
U_{n}=-\frac{d \Phi(B)}{d t}=-\frac{d}{d t} \int_{S} B_{z} d S
$$

with the result that the electric field $E_{z}$ along the beam axis would show a sinusoidal profile too, mismatching the most efficient case of a flat $E_{z}$ distribution along the axis. In the IH cavities for instance, large undercuts are used in the girder at the cavity ends: in this way the short circuit action of the end walls with respect to the $H$ mode is suppressed if the undercuts are properly matched. The original $H_{111}$ mode is transformed then into a "zero" mode $H_{11(0)}$.

In a room temperature CH-DTL it is preferable to avoid the use of a girder inside the cavity since its welding could strongly deform the outer cylinder. A different tuning strategy must be used to decrease the resonance frequency at the cavity ends. In four-vane RFQ's, which are also $H_{21(0)}$ structures, vane undercuts are used for resonant end tuning [38]. A major difference with respect to the CH-DTL is the capacitive load per unit length which is typically higher by a factor 4-6 for the RFQ. Resonant end tuning for $\mathrm{CH}$ cavities is possible by a last half drift tube with adequate length and diameter [39]. The field distribution and geometry at the $\mathrm{CH}$ cavity ends becomes then similar to reentrant cavities. In this way not only the end cell is made resonant but, at the same time, it becomes large enough to partially host the magnetic element needed for beam focusing.

In this way, very compact structures can be developed with shorter distances between adjacent tanks. The longitudinal beam dynamics, which profits from short intertank sections, favors such a design too. The dependence of the field distribution and of the resonance frequency from the length of the last half drift tube are shown in Figs. 15 and 17, respectively.

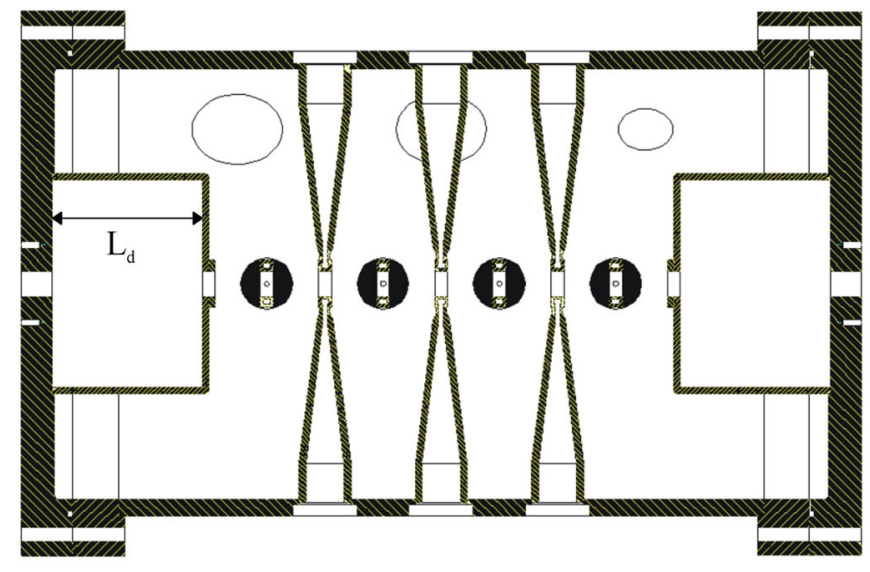

FIG. 15. The influence of the last half drift tube length $\left(L_{d}\right.$ in Fig. 16) on the field distribution on the cavity axis.

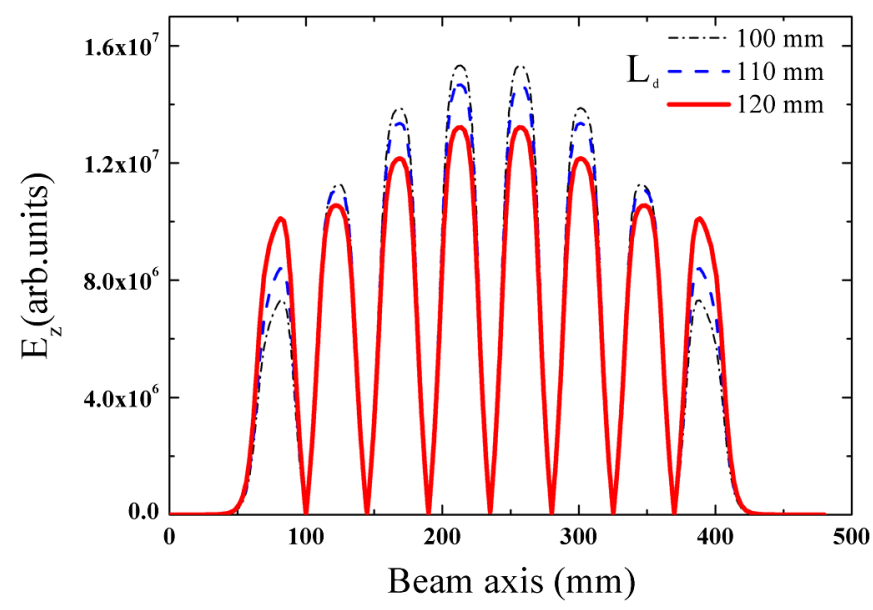

FIG. 16. A cross sectional view section of the $\mathrm{CH}$ test model. 


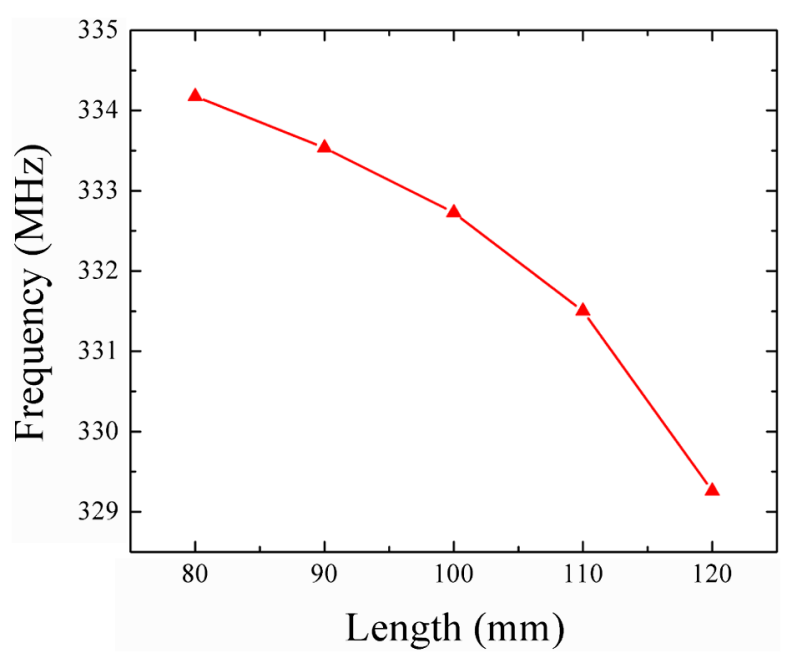

FIG. 17. The influence of the last half drift tube length on the resonance frequency for a fixed outer tank radius.

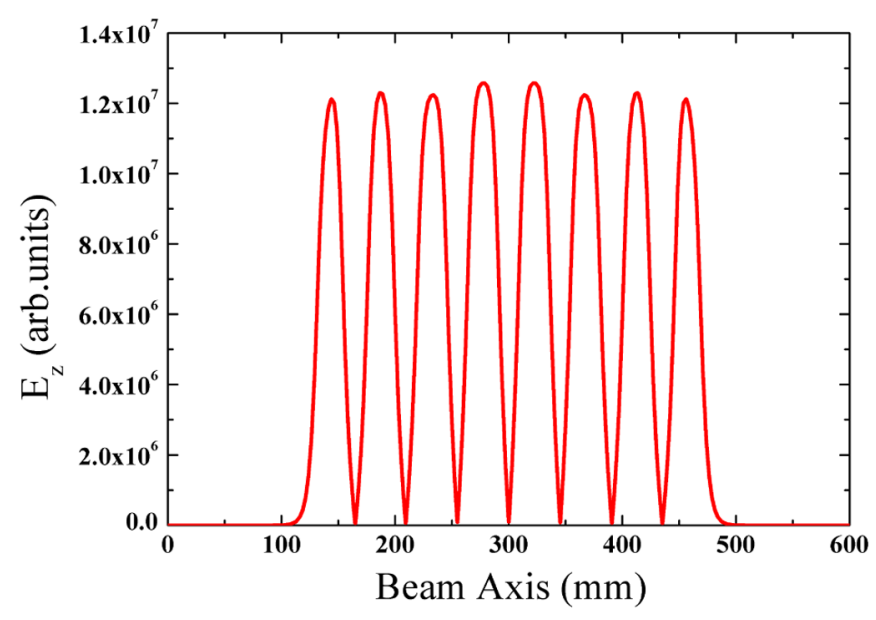

FIG. 18. The electric field distribution on axis after tuning.

However, a disadvantage is that some rf power is lost as a consequence of the capacitance between the last stem and the last half drift tube. For this reason it could become wise to remain below the "zero mode" and to make the final tuning by changing the $g / L$ ratio by locally modifying the length of the drift tubes. Generally, variation up to $\pm 20 \%$ of the local gap voltage can be achieved with this technique. In the test model, dummy drift tubes of different

TABLE I. A comparison of the of parameters for the test model.

\begin{tabular}{lc}
\hline \hline Beam aperture $[\mathrm{mm}]$ & 20 \\
\hline Frequency $[\mathrm{MHz}]$ & 326.06 \\
Closest mode $[\mathrm{MHz}]$ & 339.7 \\
$Q_{0}$ value & 13900 \\
Shunt impedance $[\mathrm{M} \Omega / \mathrm{m}]$ & 129 \\
$\beta[\%]$ & 8.255 \\
Axial voltage $V_{0}[\mathrm{MV}]$ for 1 J stored energy & 2.62 \\
\hline \hline
\end{tabular}

lengths were inserted inside the stem to tune the electric field distribution along the beam axis. In our case the $\mathrm{g} / \mathrm{L}$ ratio was varied from 0.44 , in the end cell, to 0.54 in the central ones. The final field distribution is shown in Fig. 18. Finally, Table I summarizes the main rf properties of this test model. A complementary method to reduce the capacitive load between the stem and the last half drift tube was developed in a later moment and will be described in Sec. V B

\section{Testing}

After production, assembly, and welding at Frankfurt University, the cavity was copper plated at the galvanic workshop of GSI. The thickness of the copper layer was estimated in $\approx 30 \mu \mathrm{m}$ against a skin depth of $3.6 \mu \mathrm{m}$ at $340 \mathrm{MHz}$. The success of copper plating can be considered as a milestone in the development of room temperature CH-DTL's since the feasibility of this technique was a major concern due to the complicated crossbar geometry.

After removing the dummy drift tubes, a high power test was performed under $\mathrm{cw}$ operation. The experimental setup is shown in Fig. 19. The conditioning time to achieve the maximum available power of $2 \mathrm{~kW}$ was less than 20 min without any significant multipacting effect. The cavity was operated at this high power level for some hours and no significant change in resonance frequency and in performance was observed. The absence of any frequency shift confirmed the effectiveness of the cooling system provided by the crossbar geometry.

Figure 20 shows the measured frequency spectrum including the first two modes which occur at 340 and $350 \mathrm{MHz}$ against calculated values of 338.35 and $348 \mathrm{MHz}$, respectively. The shift in frequency is due to the absence of the dummy drift tubes inserts. This large distance ensures a robust and safe operation.

The successful construction and testing of this small cavity can be considered as a major achievement in the development of the room temperature CH-DTL. In particular, the feasibility of the design on terms of construction was demonstrated and it is now proven that the copper

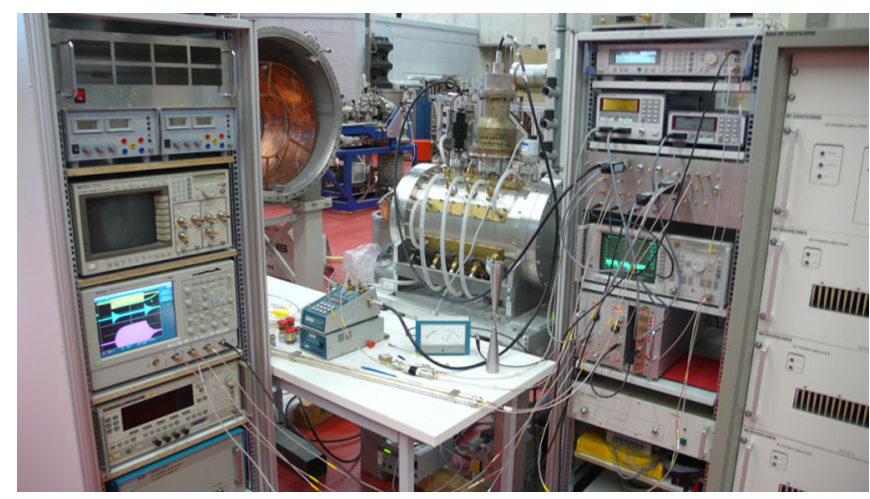

FIG. 19. The experimental setup for the high power test. The cooling system of the cavity can be clearly seen. 


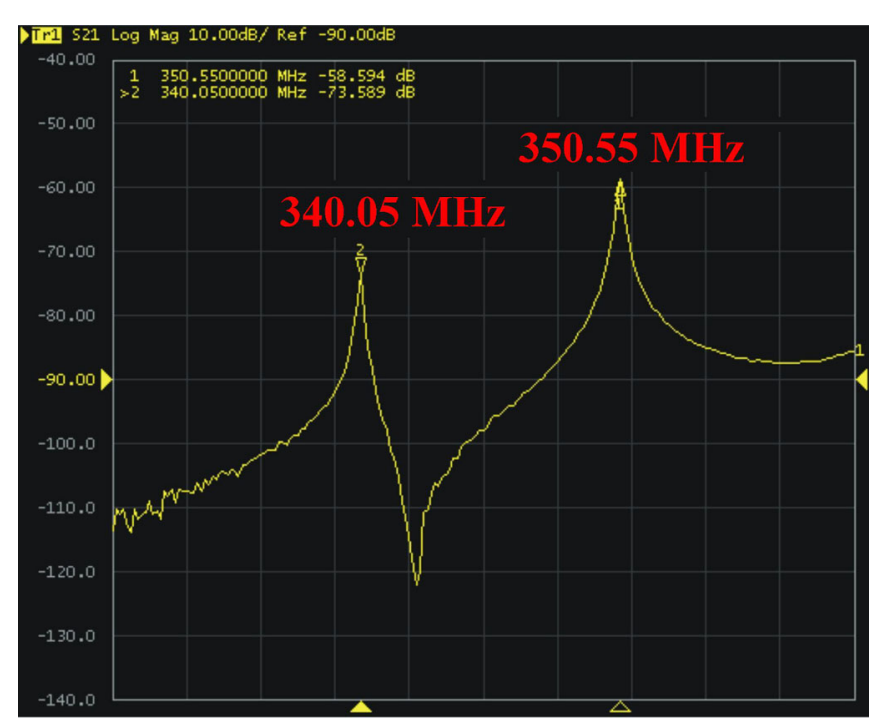

FIG. 20. The measured frequency spectrum showing the first two resonant modes.

plating technique can be applied to that new geometry. The water cooling design acts particularly efficiently opening new perspectives towards high duty cycle operation. The strategy to use dummy drift tube inserts to tune the field distribution is particularly useful too. Different configurations of the $\mathrm{g} / \mathrm{L}$ can be tested and finally the tube length can be defined accordingly to the resulting electric field distribution. At that point the exact 3D contour of the cavity is finally defined and the construction process can be initiated.

\section{THE COUPLED CH-DTL}

The 3 MW $324 \mathrm{MHz}$ klystron developed for JPARC opened new perspectives concerning the optimization of the CH-DTL. This is especially true at low energy, where

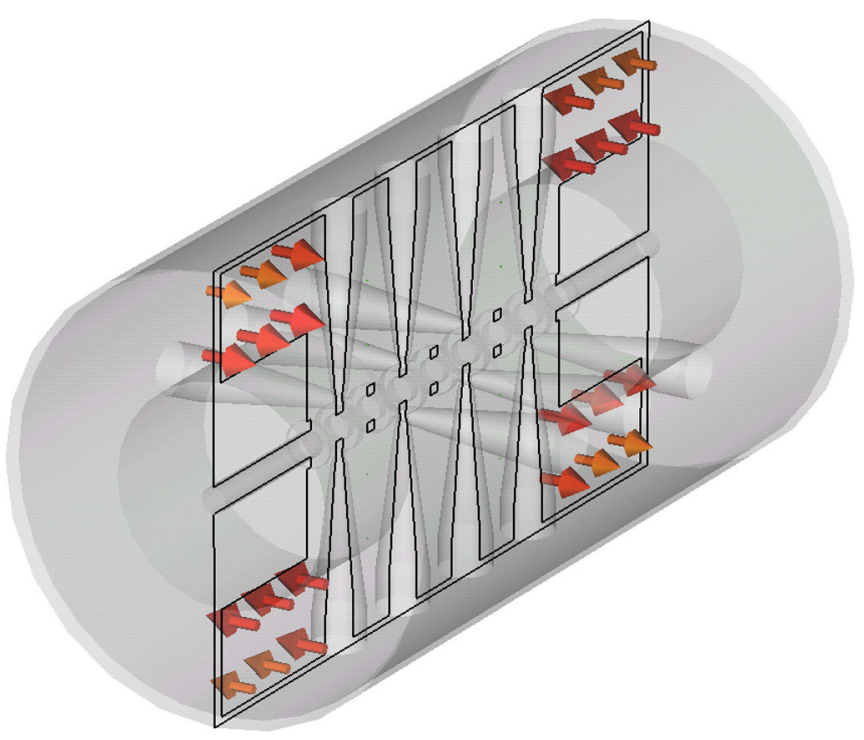

FIG. 21. Schematic view of the magnetic field orientation around the end cell. the KONUS beam dynamics requires relatively short sections, but the high shunt impedance allows one to increase the gap numbers driven by one klystron. To profit from the high shunt impedance at low energies, an innovative coupling scheme has been developed $[39,40]$ which allows one to match one klystron to two $\mathrm{CH}$ sections.

Figure 21 shows a schematic view of the magnetic field across the end cell for a CH-DTL with the last half drift tubes as described in the previous section. In this area, the magnetic field provides the required field loops $B_{\text {phi }}$ close to the cavity wall. A very similar path for the magnetic field can be observed around the drift tubes in $E_{010}$ mode cavities. Then, by a proper modification of the end cell geometry, one could expect that a transition between the $H$ and the $E$ mode could occur in that area. This transition can

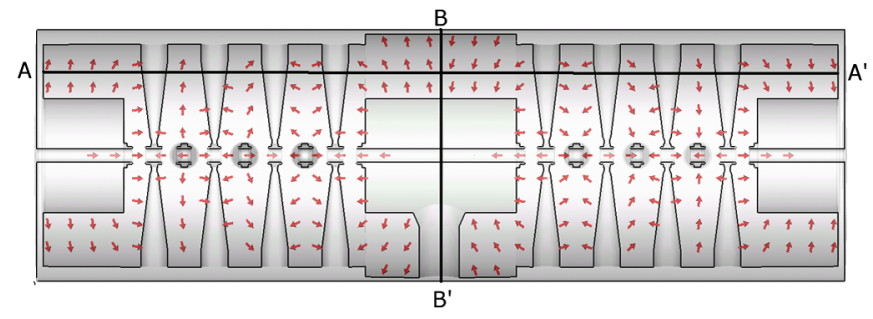

FIG. 22. The electric field pattern along the symmetry plane for the coupled CH-DTL.

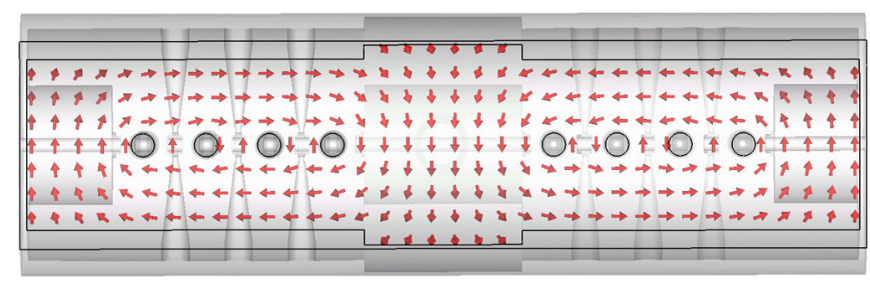

FIG. 23. The magnetic field distribution along the cut $A-A^{\prime}$ of Fig. 22.

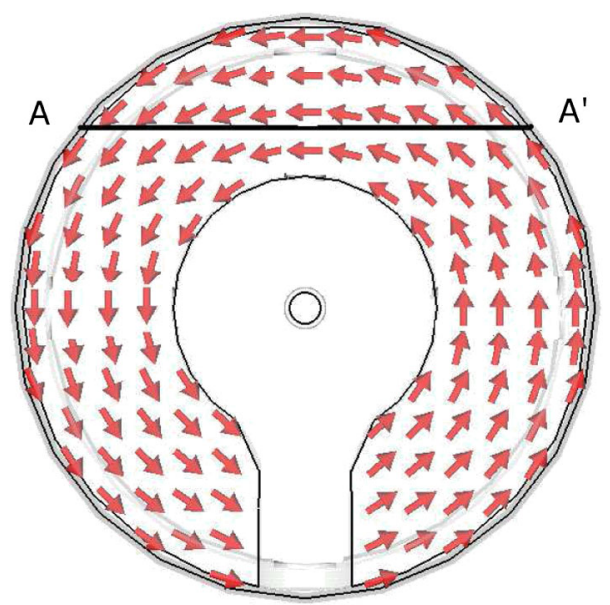

FIG. 24. The magnetic field distribution in the middle plane of the coupling cell, projection B-B' of Fig. 22. 


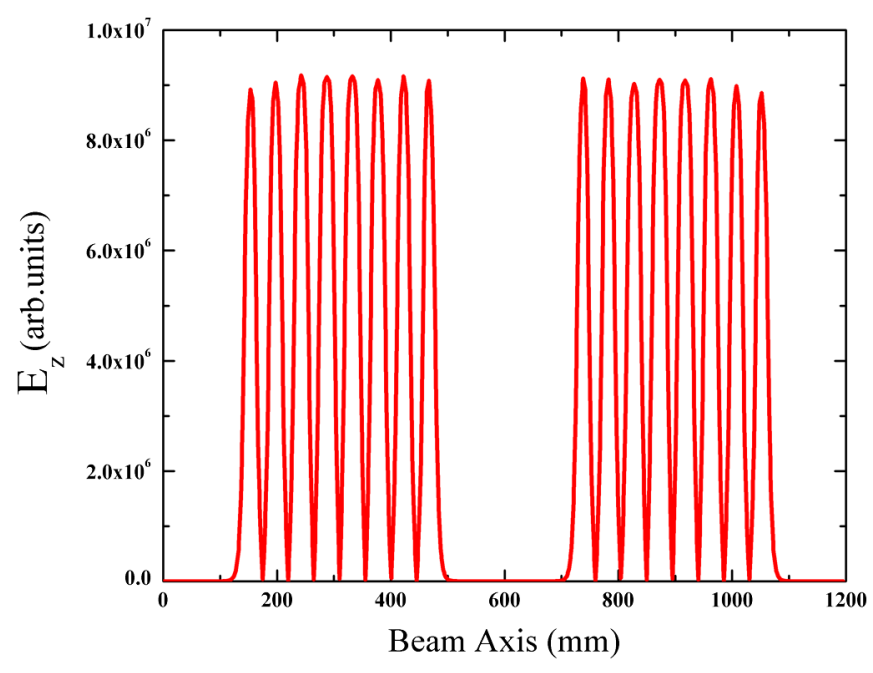

FIG. 25. The electric field distribution for the zero mode.

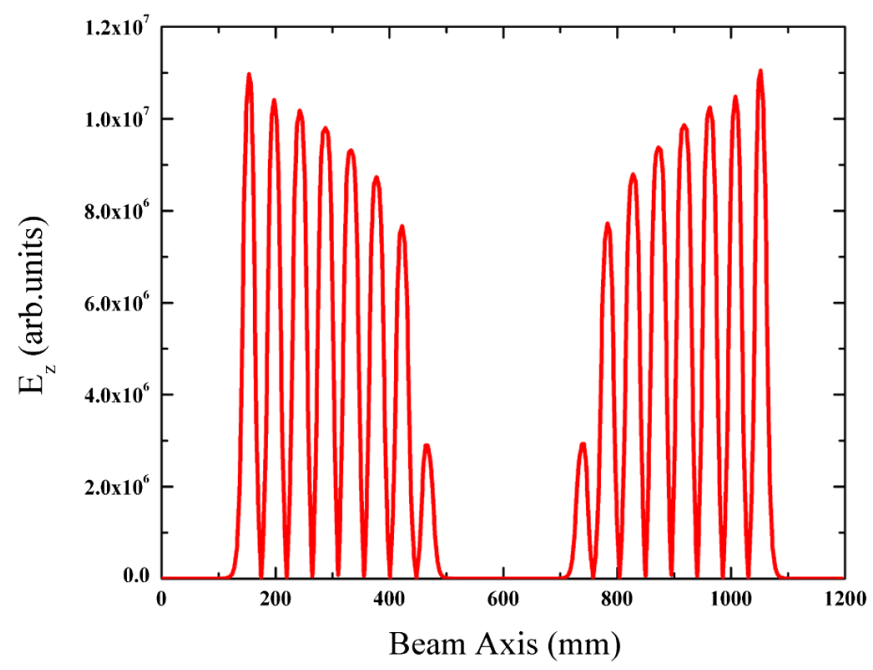

FIG. 26. The electric field distribution for the $\pi / 2$ mode.

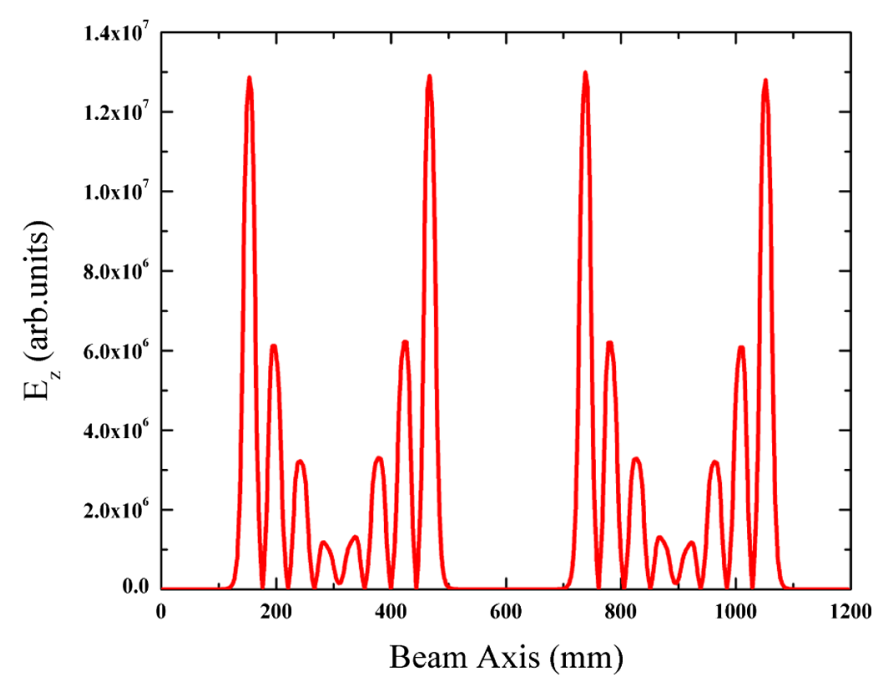

FIG. 27. The electric field distribution for the coupled $\pi$ mode.

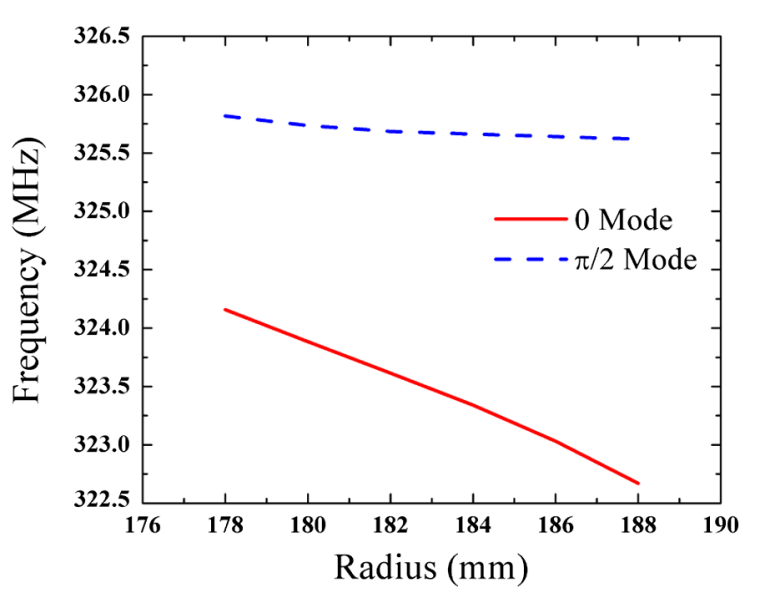

FIG. 28. The frequency of the two first resonating modes as a function of the radius of the coupling cell.

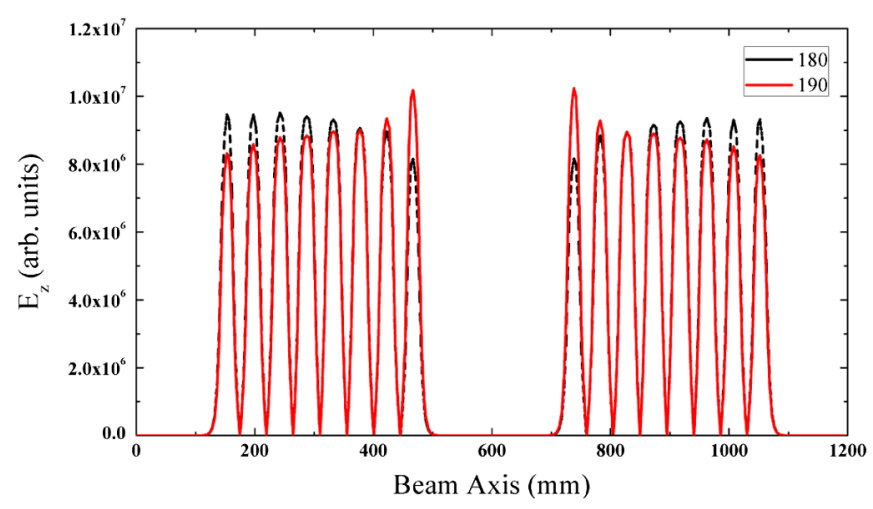

FIG. 29. The dependence of the field distribution as a function of the radius of the coupling cell.

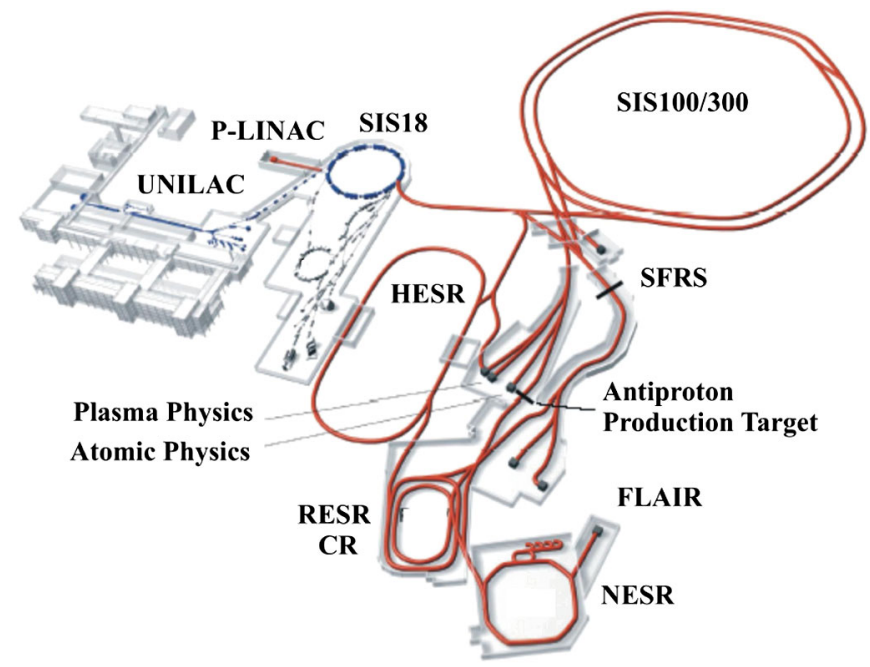

FIG. 30. Layout of the existing GSI facility and the future FAIR accelerator complex. 
TABLE II. Main parameters of the FAIR proton injector.

\begin{tabular}{lc}
\hline \hline Energy [MeV] & 70 \\
rf frequency $[\mathrm{MHz}]$ & 325.244 \\
Design current $[\mathrm{mA}]$ & 70 \\
Operation current $[\mathrm{mA}]$ & 35 \\
rf pulse $[\mu \mathrm{s}]$ & 70 \\
Repetition rate $[\mathrm{Hz}]$ & 4 \\
Duty factor $[\%]$ & 0.028 \\
Beam pulse $[\mu \mathrm{s}]$ & 35 \\
Normalized brilliance $[\mathrm{mA} / \mu \mathrm{m}]$ & $\geq 16.5$ \\
Relative momentum spread $[\%]$ & $\leq 0.1 \%$ \\
\hline \hline
\end{tabular}

be then used to couple two $\mathrm{CH}$ cavities by means of an intertank cell resonating in the $E$ mode.

For a better illustration of the concept, one could think to mirror the cavity shown in Fig. 21 at an end wall and then replacing that wall by a stem supporting the resulting central large drift tube. That geometry leads to a scheme where two $\mathrm{CH}$ sections are connected through an intertank section which houses a magnetic triplet. The coupling is created by adjusting the radius of the intertank cell so that the resonance frequency of this unit matches the adjacent $\mathrm{CH}$ sections. The rf simulations have proven that an effective coupling is achieved with this geometry as shown from Figs. 22-24. In particular, Fig. 24 shows very clearly that the intertank section oscillates in the Alvarez mode. The rf currents oscillate along the central tube, creating a 0 -mode section which connects the two CH-DTL's.

The rf synchronism with the beam is ensured by a length of the coupling cell equal to $N \cdot \beta \lambda-\Delta \phi$, where $\Delta \phi$ denotes the shift in rf phase required by the KONUS beam dynamics.

With respect to standard options for coupled linacs, this solution presents the advantage to avoid slots or dedicated coupling cavities which are difficult to be tuned. In our case an inductively acting plunger can be moved inside the coupling cell to adjust the resonance frequency and the field distribution at the adjacent cells. Field simulations were performed investigating the coupling of two $\mathrm{CH}$ sections similar to the one described in the previous section. The length of the coupling cell was $3 \beta \lambda$ for a total inner length of $1185 \mathrm{~mm}$.

The main results of those preliminary investigations are in agreement with the magnetic coupling model. Beyond the $H_{21(0)}$ mode, denoted as 0 mode (see the field distribution shown in Fig. 25), the coupling generates near adjacent modes. The $H_{211}$ mode, the so-called " $\pi / 2$ " mode is the noncoupled mode where the magnetic field is oriented in one direction along the beam axis through each of the four quadrants. This field distribution is in agreement with the theory of coupled resonators [see Eq. (4.18) in [41]], which foresees the $\pi / 2$ mode as independent from the coupling strength and with a coupling cell where no electric field is present. The amplitude of the electric field shows a zero transition through the coupling cell as one can see in Fig. 26. Finally, the $\pi$ mode represents the coupling between the two $\mathrm{CH}$ cavities oscillating at the next longitudinal $\mathrm{H}_{212}$ mode (Fig. 27).

The strength of the coupling can be described by the coupling factor [42]

$$
k \approx \frac{\omega_{\pi}-\omega_{0}}{\omega_{0}},
$$

where $\omega_{0}$ and $\omega_{\pi}$ are the frequencies of the lowest and of the highest mode, respectively. According to the theory this parameter is independent from the resonance frequency of the noncoupled mode. This result is well confirmed by simulations as shown in Fig. 28. The resonance frequency of the coupling mode is a decreasing function of the radius of the coupling cell while the dependence of the resonance of the $\pi / 2$ mode against this parameter is very weak. On the other hand, if the distance between the modes is increased, this must also correspond to an increase of the coupling strength, which is also confirmed by simulations. As shown in Fig. 29, as the radius of the coupling cell is increased, the field in the adjacent cells close to the intertank section becomes stronger increasing the strength of the coupling.

This phenomenon should be taken into account since higher voltage levels in this area could increase the risk of sparking between the lens and the adjacent stems of the $\mathrm{CH}$ structures, as happened between the lens housing and an adjacent drift tube inside an earlier IH cavity [43].

\section{THE FAIR PROTON INJECTOR}

The FAIR Project [44] (Facility for Antiprotons and Ions Research), sketched in Fig. 30, is the new international facility under development at GSI, Darmstadt, Germany. The heart of FAIR consists of a superconducting double synchrotron, the SIS 100/300, with magnetic rigidities of 100 and $300 \mathrm{Tm}$, respectively. The present GSI facility will serve as an injector chain after the consistent upgrade [45] which is required for the FAIR operation in terms of beam intensity and quality.

For instance, the antiproton physics program demands a primary proton beam of $2 \times 10^{16} \mathrm{p} / \mathrm{h}$, which is way beyond the capabilities of the existing UNILAC. For this reason a new linac has to be designed as a dedicated

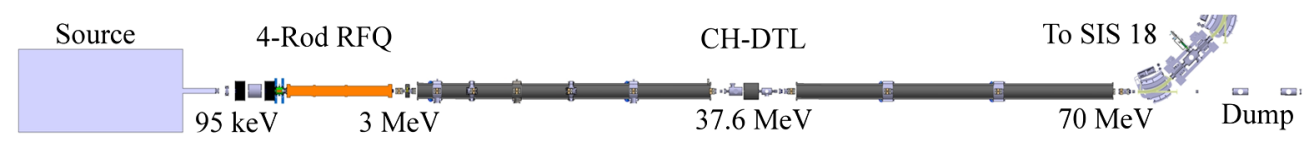

FIG. 31. The schematic view of the FAIR proton injector. 
TABLE III. Main parameters of the $\mathrm{CH}$ cavities for the FAIR proton injector.

\begin{tabular}{lccccc}
\hline \hline Cavity & Coupling & Energy $[\mathrm{MeV}]$ & $\varnothing_{i}[\mathrm{~mm}]$ & Gaps & Length $[\mathrm{m}]$ \\
\hline 1 & Yes & $3.0-11.7$ & 20 & 22 & 1.7 \\
2 & Yes & $11.7-24.1$ & 20 & 27 & 2.7 \\
3 & Yes & $24.1-37.6$ & 20 & 32 & 4.0 \\
4 & No & $37.6-48.1$ & $20-24$ & 20 & 2.9 \\
5 & No & $48.1-59.3$ & $20-24$ & 21 & 3.1 \\
6 & No & $59.3-70.0$ & $20-24$ & 21 & 3.4 \\
\hline \hline
\end{tabular}

injector for the SIS 18 synchrotron. The final energy of $70 \mathrm{MeV}$ allows the saturation of $\bar{p}$ production [46] while the multiturns injection scheme requires at least $35 \mathrm{~mA}$ within a normalized brilliance of $16.5 \mathrm{~mA} / \mu \mathrm{m}$. The main parameters of this new injector are summerized in Table II.

The choice of the frequency in this energy range is a compromise between the requirements of minimized radial defocusing at low energy,

$$
\Delta p_{r} \propto \frac{f}{(\beta \gamma)^{2}},
$$
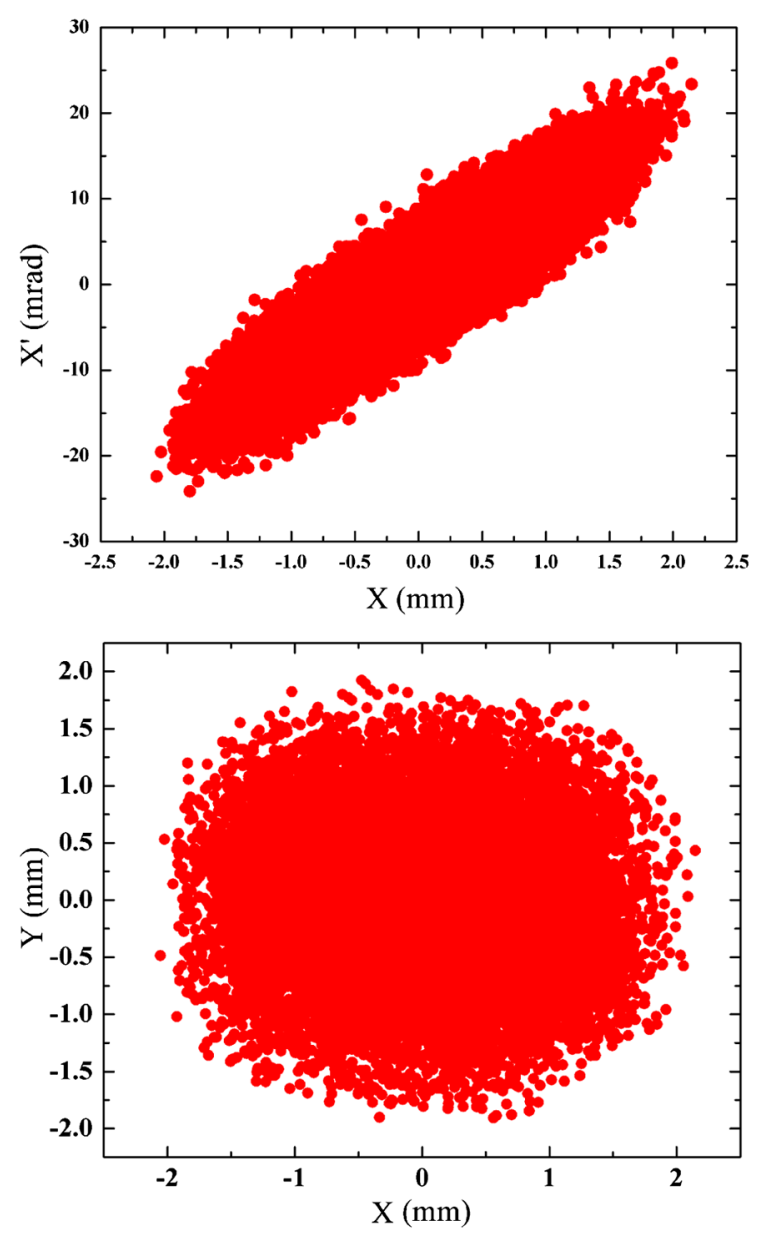

and the optimization of the rf efficiency,

$$
Z T^{2} \propto \sqrt{f} .
$$

A good compromise results to be the range between 300 and $400 \mathrm{MHz}$ where adequate amplifiers are available. For the GSI proton injector the value of $325.224 \mathrm{MHz}$ has been chosen: this value corresponds to 9 times the basic resonance frequency of the existing high current injector of the UNILAC (36.136 MHz) and allows the use of commercial 3 MW klystrons.

The scheme of the FAIR proton injector is sketched in Fig. 31: an electron-cyclotron-resonance ion source will deliver the protons at an energy of $95 \mathrm{keV}$. The beam is first accelerated by a four-rod RFQ [47] to the energy of $3 \mathrm{MeV}$. After the matching section, consisting of a quadrupole triplet, an rf buncher, and a doublet, the beam enters the first section of the drift tube linac composed of three pairs of coupled CH-DTL's. At the energy of $37 \mathrm{MeV}$, a $1.6 \mathrm{~m}$ long dedicated diagnostic section is foreseen. At this energy space charge effects are reduced and $\mathrm{rf}$ defocusing is of minor concern. Since the KONUS beam dynamics allows for longer lens-free sections, it was decided to base the high energy section on standard lens-free $\mathrm{CH}$
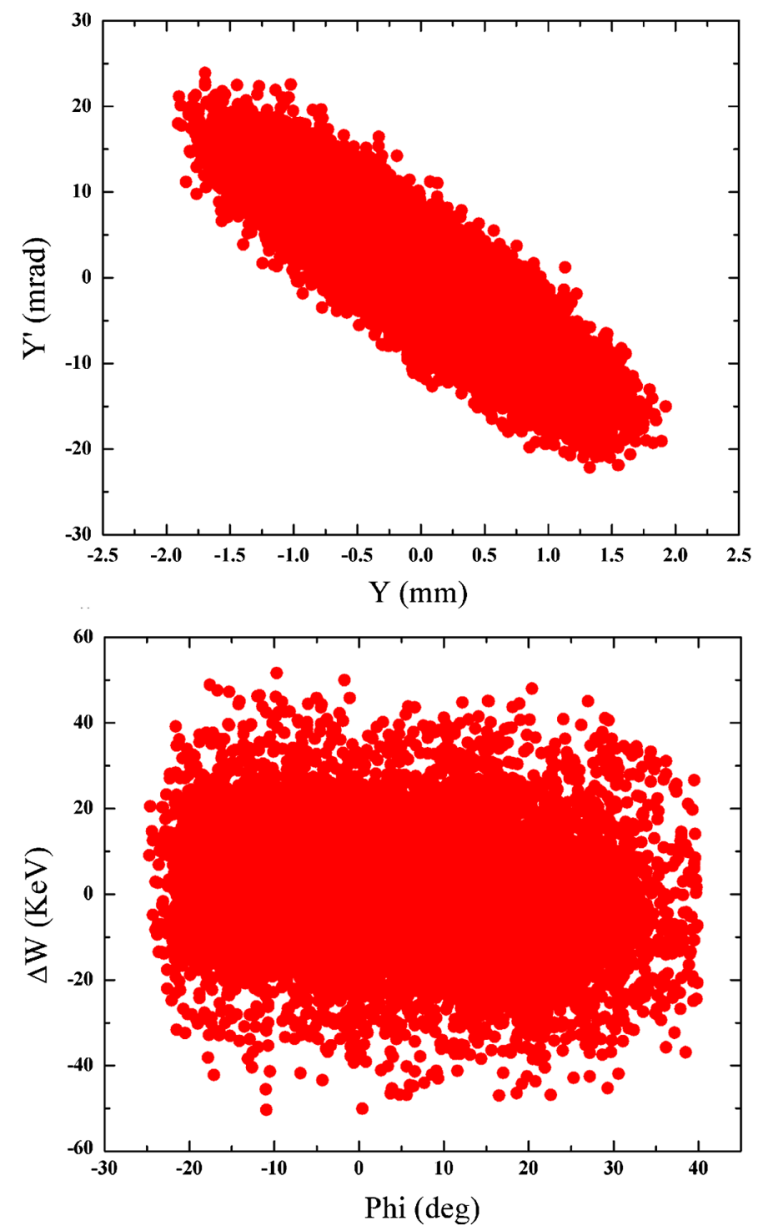

FIG. 32. The particle distribution at the exit of the RFQ. 
cavities with slightly larger beam aperture at each cavity end. In this way the number of quadrupole triplets is reduced while the general simplification of the mechanical and $\mathrm{rf}$ design results in an effective cost saving. The rf simulations showed that energy gains up to $11.2 \mathrm{MeV}$ along 21 gaps can be achieved within a maximum rf power demand of $950 \mathrm{~kW}$ per individual $\mathrm{CH}$ cavity. At the energy of $70 \mathrm{MeV}$, the protons are injected into the existing transfer channel which connects the UNILAC to the SIS 18.

The main $\mathrm{CH}$ cavity parameters of the proton linac are summarized in Table III.

\section{A. Beam dynamics}

Beam dynamics simulations were performed within a wide range of input currents. The ion source can in fact deliver a maximum current of $100 \mathrm{~mA}$ while RFQ tracking performed with TOUTATIS [48] and PARMTEQM [49] has shown a transmission higher than $97 \%$. RFQ simulations were performed with up to 100000 macroparticles but around 20000 were used for the design of the DTL section.

The particle distribution at the exit of the RFQ is shown in Fig. 32.

The main linac was designed assuming an RFQ output current ranging from 45 to $70 \mathrm{~mA}[50,51]$ using the LORASR code [52]. Even if $35 \mathrm{~mA}$ are required for the $\bar{p}$ program, it is realistic to expect in the future an upgrade of the cooling capabilities of the FAIR cooling ring $C R$. In this case the intensity of the primary proton beam should be consequently increased. Moreover, the choice of $70 \mathrm{~mA}$
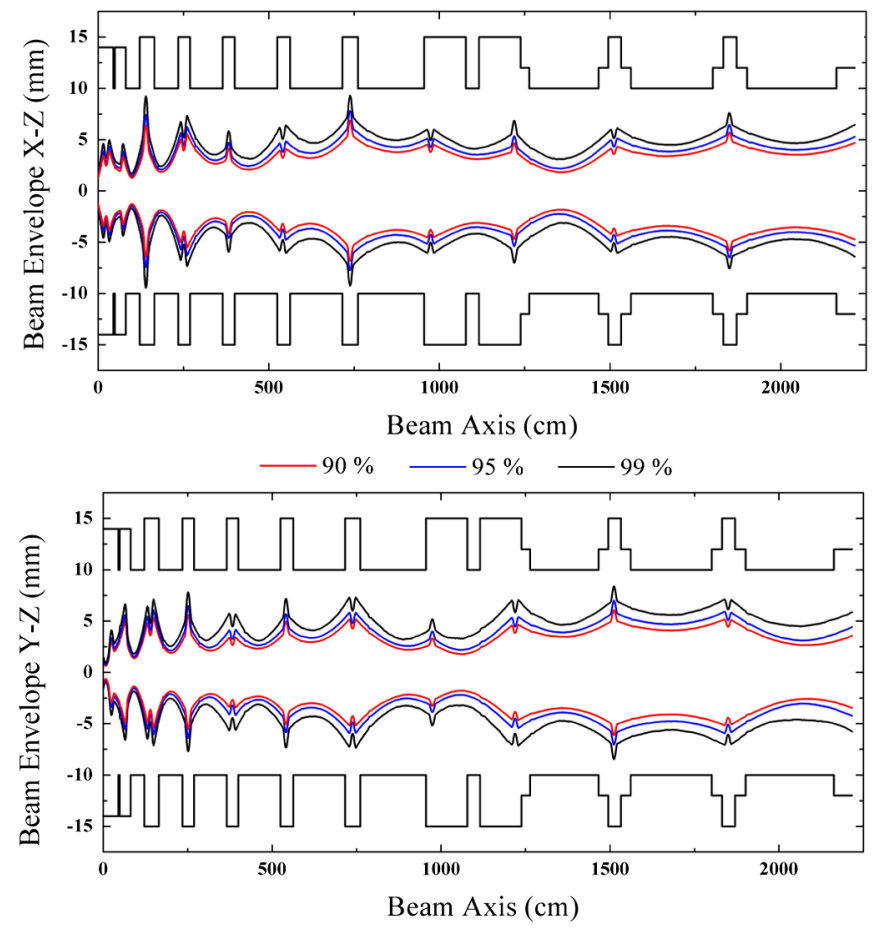

FIG. 33. The transverse beam envelopes of the FAIR proton injector.

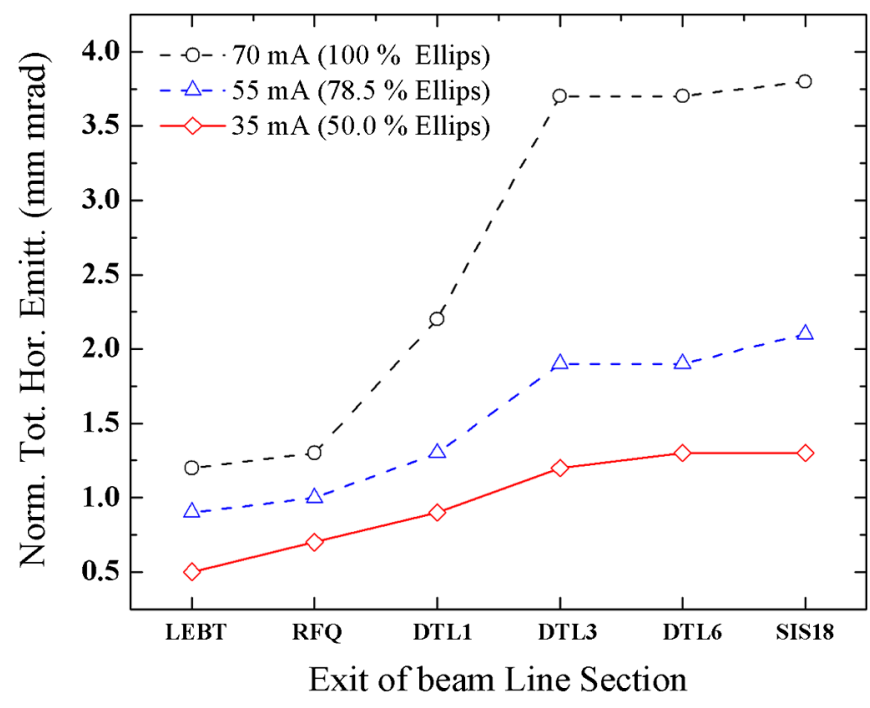

FIG. 34. The development of the emittance growth when $70 \mathrm{~mA}$ are injected from the RFQ. The three lines represent the growth of the emittances including $50 \%, 78 \%$, and $100 \%$ of the beam intensity.

as design current results in a safety margin against beam losses and does not exceed the available power from the klystrons.

The beam envelopes for the design current of $70 \mathrm{~mA}$ are shown in Fig. 33, a robust safety margin against beam losses is available. Particularly important is the development of the emittance growth along the beam line. This

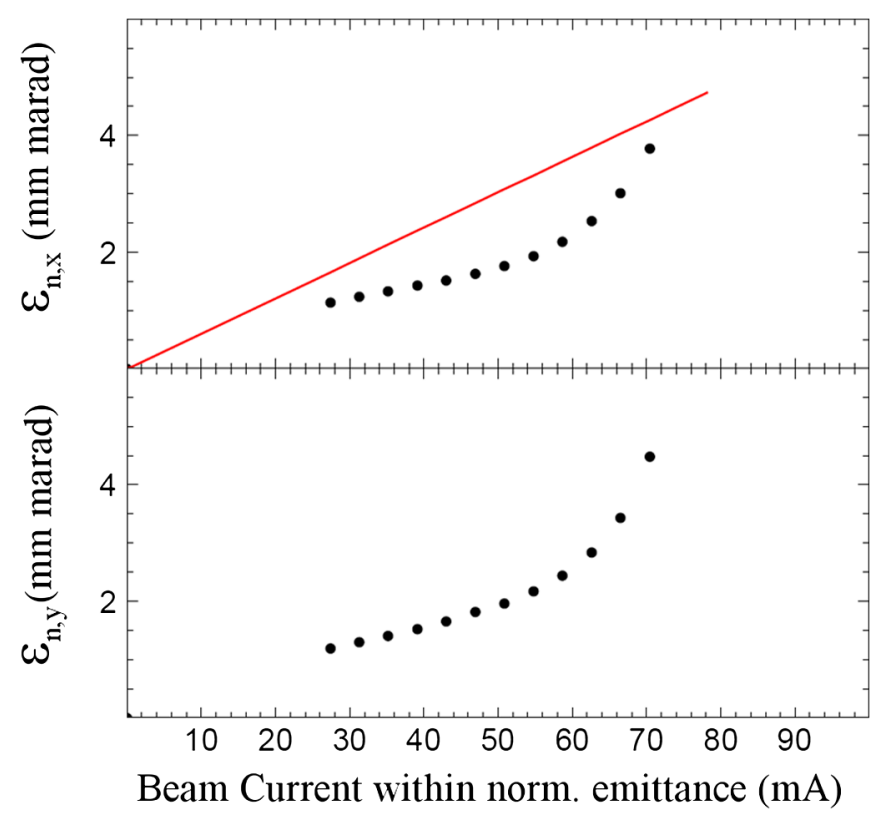

FIG. 35. The dependence of the normalized emittance at the linac exit as a function of the correspondent beam current (dots). The red line represents the upper limit for the foreseen multiturn injection scheme. 
parameter is in fact the main constraint to be respected for the horizontal multiturn injection into the SIS18.

Figure 34 shows the emittances corresponding to 70, 55, and $35 \mathrm{~mA}$ when a full $70 \mathrm{~mA}$ beam from the RFQ is injected into the DTL section. The three lines show the emittance growth for the corresponding ellipses in the horizontal phase space which includes a current of 35, 55 , and $70 \mathrm{~mA}$, respectively. Figure 35 presents the emittance including the current stated along the abscissa at the injection point. The red curve is the upper limit for the emittance that the injector, at given current, must provide. This limit is obtained from the foreseen multiturn scenario and it depends mainly on several synchrotron parameters, such as circumference, injection energy, acceptance, and filling factor. For a proper injection the linac emittance must remain below the red curve.

This brilliance analysis [53] shows that the best window for the injection into the synchrotron occurs between 50 and $60 \mathrm{~mA}$, where also emittance growth is limited (Fig. 35).

Finally, Fig. 36 shows the beam distribution after the last $\mathrm{CH}$ cavity. In Table IV the main parameters of the FAIR proton injector are listed for a beam current of $70 \mathrm{~mA}$.
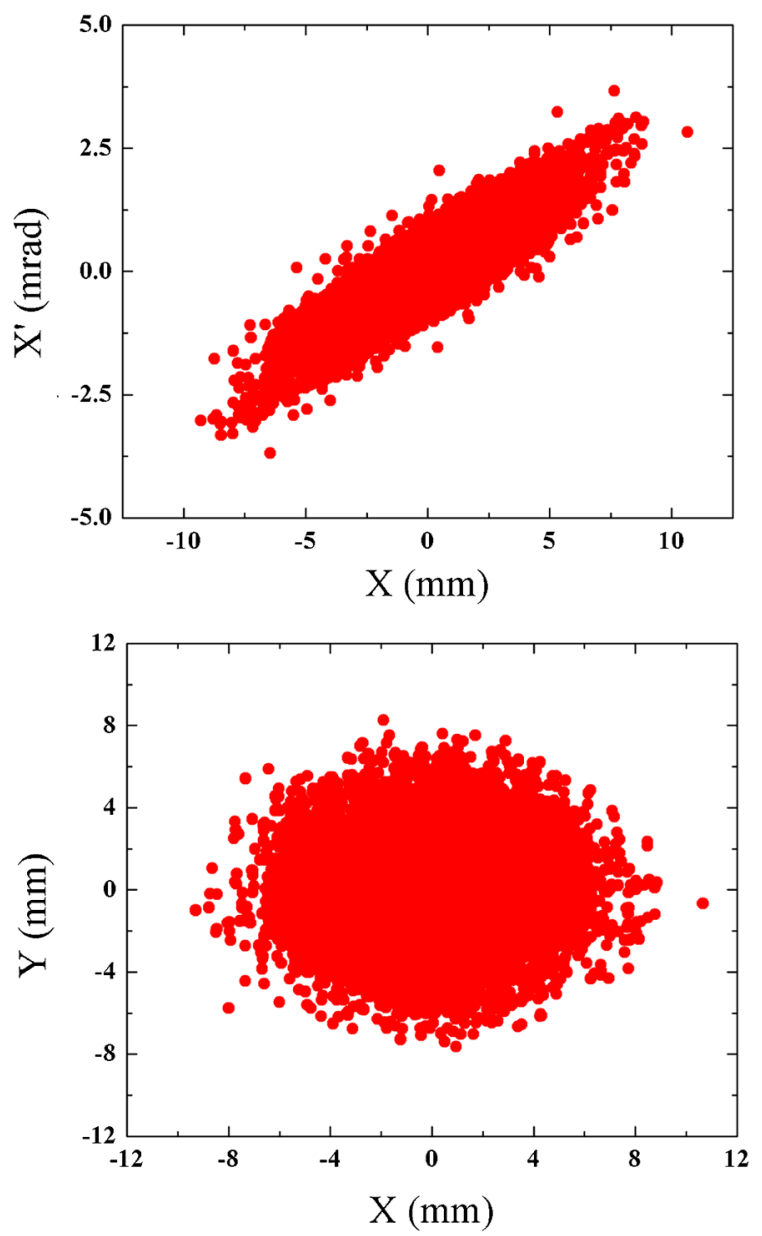

TABLE IV. Beam evolution of the FAIR proton injector at $70 \mathrm{~mA}$.

\begin{tabular}{|c|c|}
\hline RFQ output $100 \% \mathrm{rms} X-X^{\prime}$, normalized (mm mrad) & 0.23 \\
\hline RFQ output $100 \% \mathrm{rms} Y-Y^{\prime}$, normalized (mm mrad) & 0.21 \\
\hline RFQ output $100 \%$ rms $\Delta \mathrm{W}-\Phi(\mathrm{KeV} n s)$ & 1.41 \\
\hline RFQ output $95 \% \in X-X^{\prime}$, normalized (mm mrad) & 1.22 \\
\hline RFQ output $95 \% \in Y-Y^{\prime}$, normalized (mm mrad) & 1.14 \\
\hline RFQ output $95 \% \epsilon \Delta \mathrm{W}-\Phi(\mathrm{keV} \mathrm{ns})$ & 9.01 \\
\hline DTL output $100 \% \mathrm{rms} X-X^{\prime}$, normalized (mm mrad) & 0.43 \\
\hline DTL output $100 \% \mathrm{rms} Y-Y^{\prime}$, normalized (mm mrad) & 0.45 \\
\hline DTL output $100 \%$ rms $\Delta \mathrm{W}-\Phi(\mathrm{KeV}$ ns $)$ & 2.65 \\
\hline DTL output $95 \% \epsilon$, normalized $X-X^{\prime}$ & 2.35 \\
\hline DTL output $95 \% \epsilon$, normalized $Y-Y^{\prime}(\mathrm{mm} \mathrm{mrad})$ & 2.68 \\
\hline DTL output $95 \% \in \Delta \mathrm{W}-\Phi(\mathrm{KeV} \mathrm{ns})$ & 17 \\
\hline Particle number & 20000 \\
\hline
\end{tabular}

\section{B. Scaled model of the second coupled CH cavity for FAIR}

The design of the low energy part of the proton injector is based on coupled $\mathrm{CH}$ cavities. In order to complete the R\&D phase of the project, a scaled model of the second cavity has been built and investigated at Frankfurt
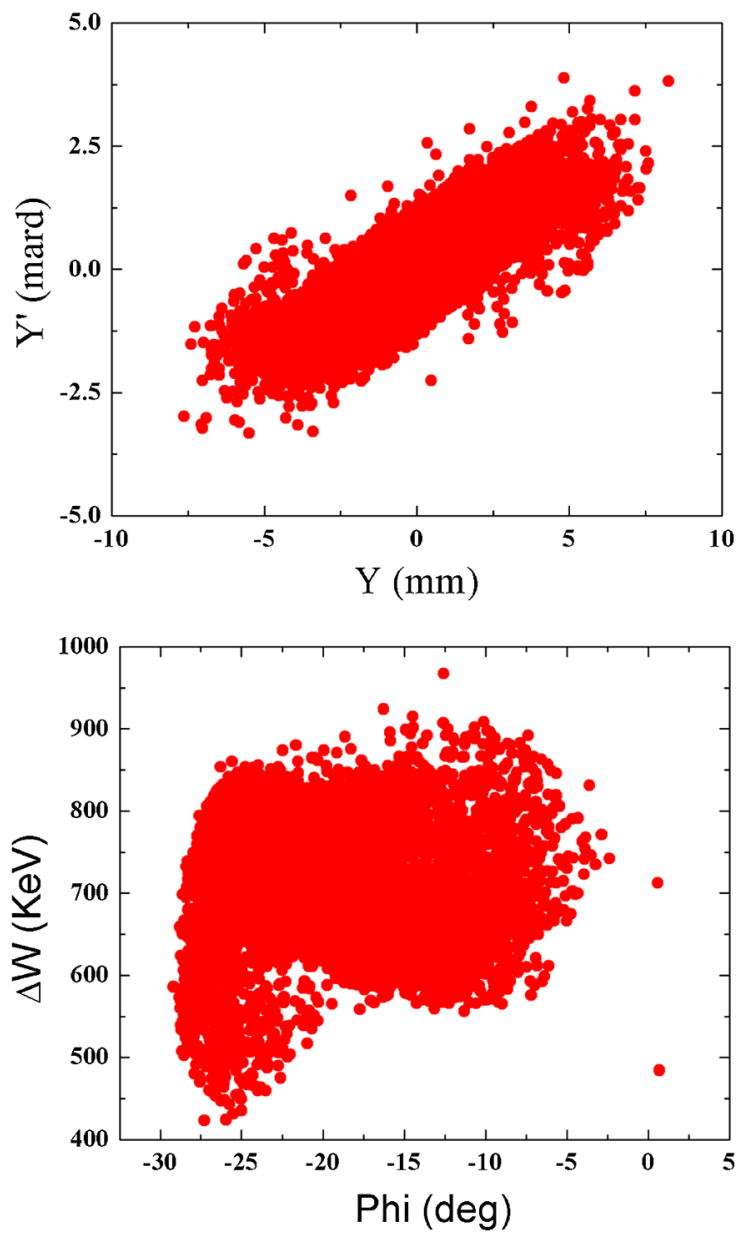

FIG. 36. The output particle distribution at the exit of the DTL section. 


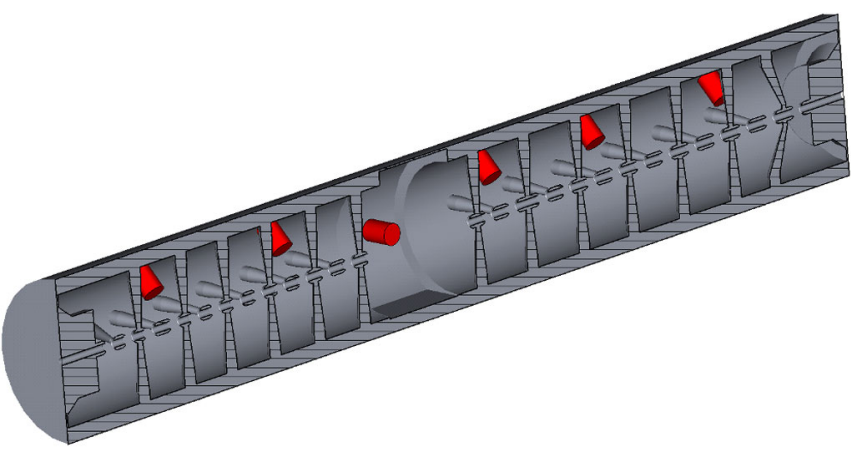

FIG. 37. Cross sectional view of cavity 2 of the GSI proton injector showing the position of the inductive tuners.

University. At present the full scale prototype is under construction. The choice to build the second coupled cavity, and not, for example, the first one, left more flexibility in the design of the front part of the linac (including the RFQ). In this way only the entrance energy of the second coupled module had to be definitely fixed.

The $2.7 \mathrm{~m}$ long prototype cavity is shown in Fig. 37. It consists of 27 gaps with an effective accelerating voltage of 13.5 MV and with a constant beam aperture of $20 \mathrm{~mm}$. The energy range is from 11.7 to $24.4 \mathrm{MeV}$ with an intermediate energy through the coupling cell occurring at $17.4 \mathrm{MeV}$. The radii of the three sections are 179,217 , and $181 \mathrm{~mm}$, respectively, while the outer radius of the lens housing is $110 \mathrm{~mm}$. With respect to the model described in Sec. III the design of the drift tubes was modified to increase the mechanical stability during welding. Table V summarizes the main properties of this coupled module.

The 1:2 scaled model reproduces the 3D contour of the real resonator and it consists of an outer cylinder made of aluminum while stems, lenses, and the end cell half drift tubes are made of brass. A further tuning concept based on an inclined stem geometry for the end cells of the cavity is

TABLE V. Main parameters of the second cavity of the FAIR proton injector. The rf parameters include a reduction safety margin of $15 \%$. The length of the coupling cell is included in the definition of $Z T^{2}$.

\begin{tabular}{lc}
\hline \hline No. of gaps & $27(13+14)$ \\
Frequency $(\mathrm{MHz})$ & 325.2 \\
Energy range $(\mathrm{MeV})$ & $11.7-24.4$ \\
Effective voltage $(\mathrm{MV})$ & 13.50 \\
$E_{0}(\mathrm{MV} / \mathrm{m})$ & 5.58 \\
Average transit time factor & 0.909 \\
$Q_{0}(13700$ \\
$Z T^{2}(\mathrm{M} \Omega / \mathrm{m})$ & 50 \\
$\mathrm{rf}$ losses $(\mathrm{MW})$ & 1.34 \\
Kilpatrick factor & 2 \\
Beam aperture $(\mathrm{mm})$ & 20 \\
Lens aperture $(\mathrm{mm})$ & 30 \\
Inner total length $(\mathrm{m})$ & 2.7 \\
\hline \hline
\end{tabular}

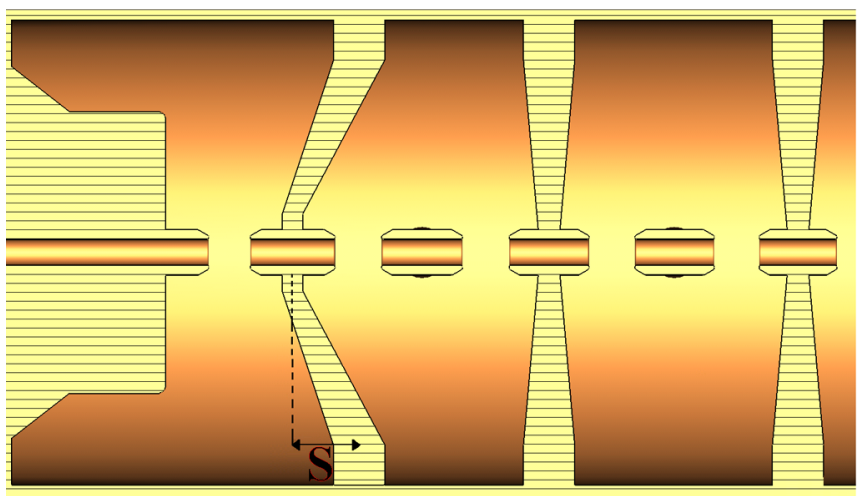

FIG. 38. A detail of the first cells of the second resonator of the FAIR proton injector showing the inclined first stem.

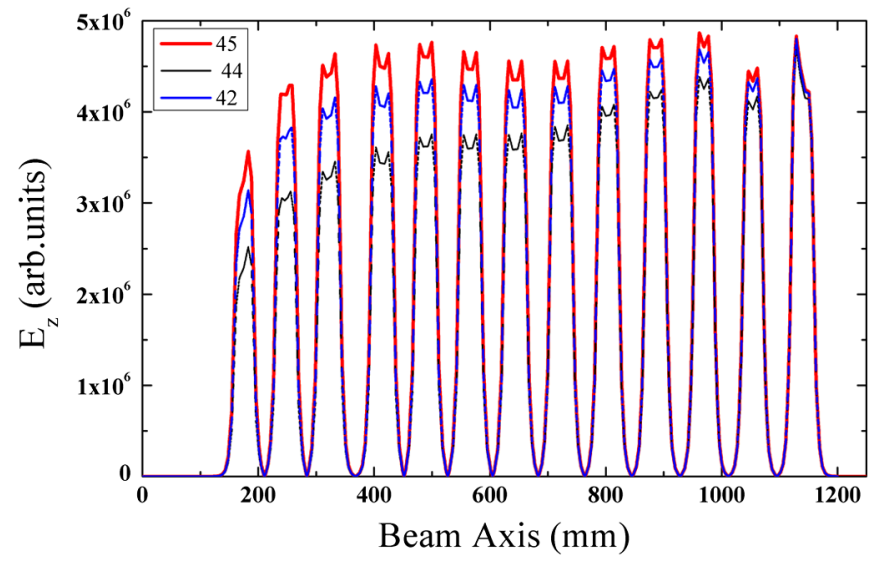

FIG. 39. Dependence of the longitudinal component of the electric field in the first section of the second FAIR resonator as a function of the inclination of the first stem (parameter $\mathbf{S}$ of Fig. 38).
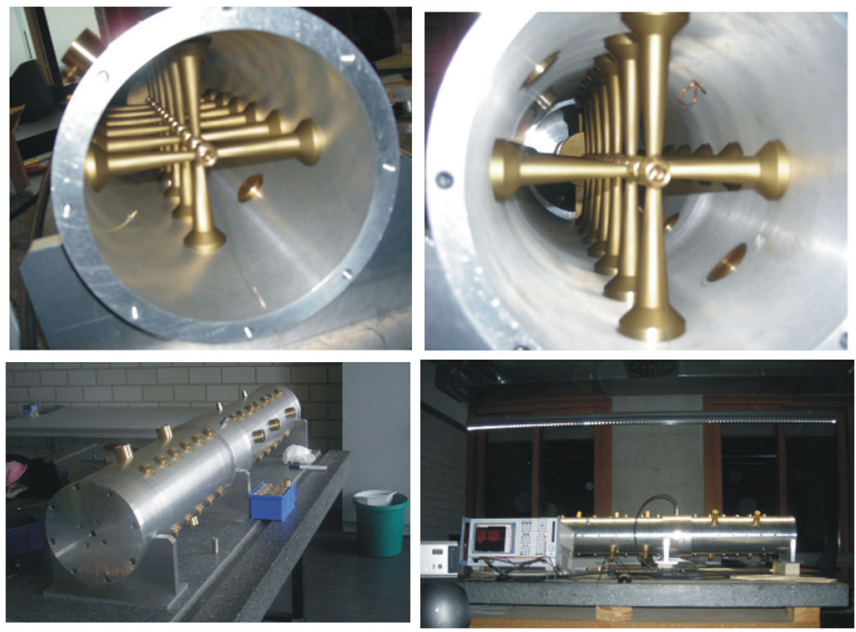

FIG. 40. Photos of the 1:2 model of the second cavity of the FAIR proton injector. 


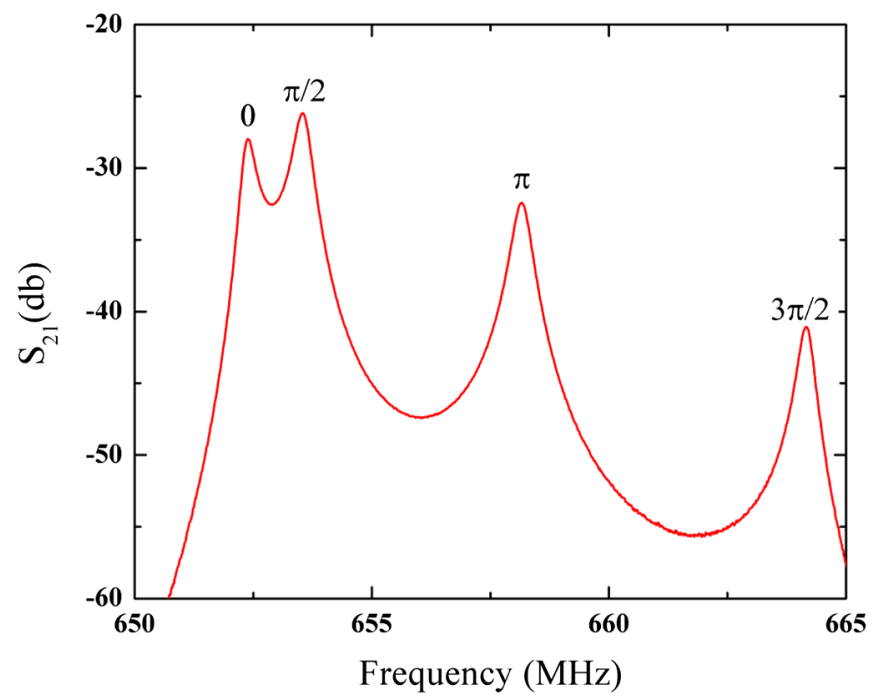

FIG. 41. The measured frequency spectrum of the 1:2 scaled model for the second resonator of the FAIR proton injector.

shown in Fig. 38. This solution allows one to increase the magnetic flux in the last cells, thus increasing the voltage, and at the same time, it reduces the capacitive load between the end stem and the last half drift tube. Moreover, the length of the last half drift tube can be reduced resulting in a strong advantage in terms of mechanical design and construction. This solution was originally developed for the superconducting CH-DTL [54] and later applied to the room temperature design of the FAIR proton injector. In particular, the dependence of the electric field in the first section of the scaled model as a function of the inclination of the stem is shown in Fig. 39. The tuning strategy is completed by 11 mobile plungers mounted along the cavity, two pairs in the first resonator, three pairs in the second one, and one located inside the coupling cell. An overview of the cavity on the test bench is shown in Fig. 40.

The measurements of the resonance frequency, presented in Fig. 41, show the accelerating mode resonating

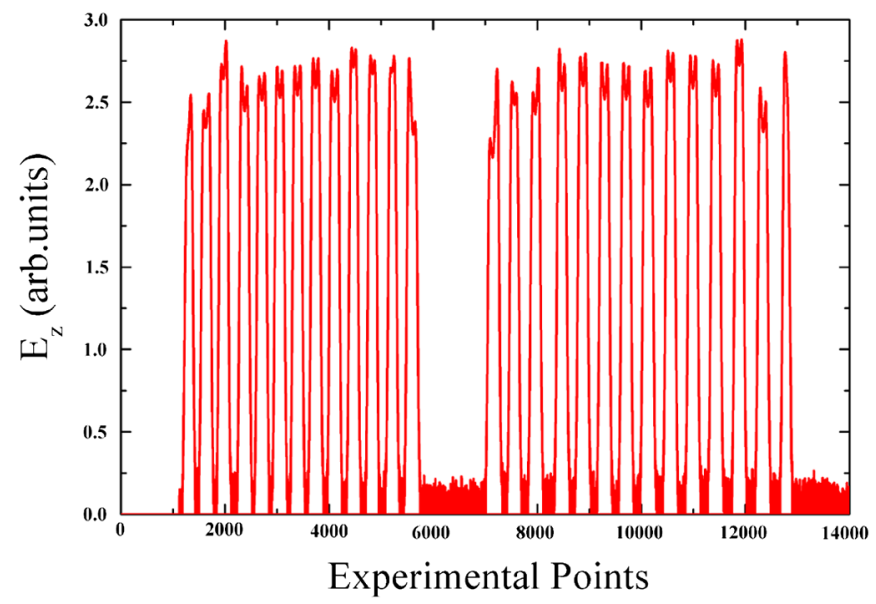

FIG. 42. The measured field distribution of the zero $H_{21(0)}$ mode.

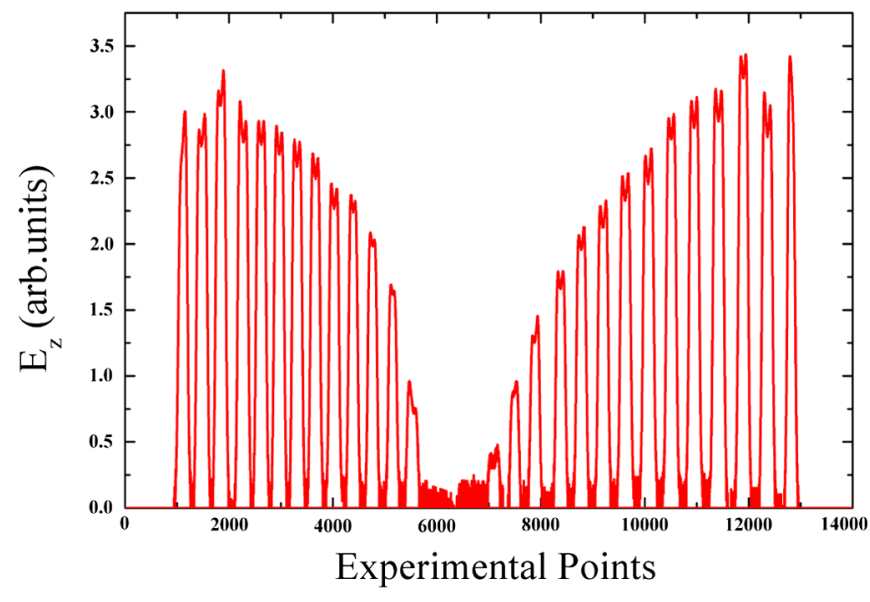

FIG. 43. The measured field distribution of the $H_{211} \pi / 2$ mode.

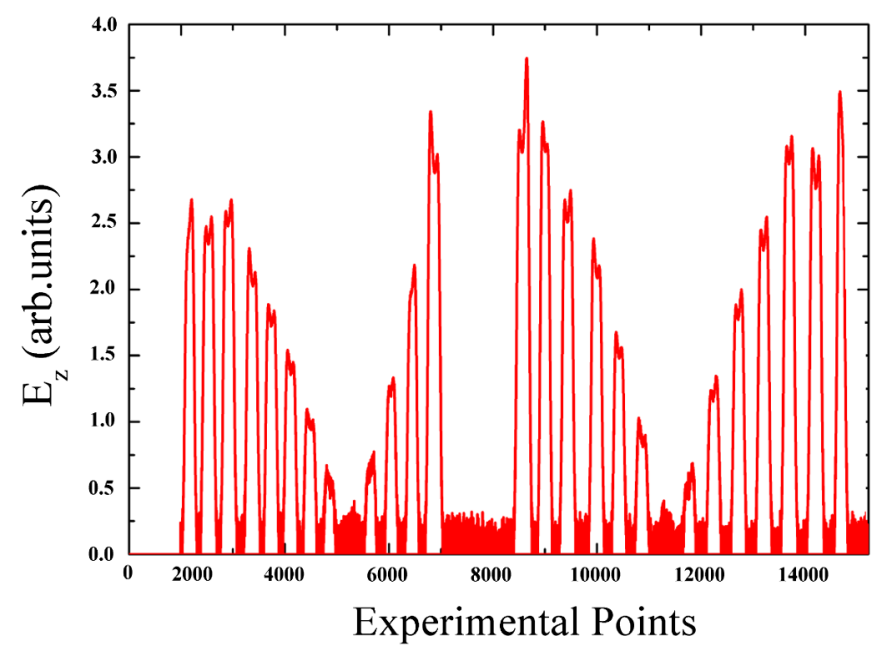

FIG. 44. The measured field distribution of the $H_{212} \pi$ mode

at $652.3 \mathrm{MHz}$ (against a simulated value of $650.5 \mathrm{MHz}$ ) with the closest higher modes occur at 653.5 and $658.2 \mathrm{MHz}$, respectively. The field distribution for the corresponding modes is represented from Figs. 42-44 and confirms the simulation results of the previous chapter. The field distribution of the zero mode was flattened by the use of the mobile tuners and the corresponding voltage distribution was in good agreement with the design values. Beam dynamics simulations shown in the previous section were actually performed by using those measured values.

The power feeder will be located in the coupling cell. In this way the $\pi / 2$ mode cannot be driven by the klystron due to the presence of the field node in that position.

\section{Mechanical layout}

Following those encouraging results, GSI and Frankfurt University agreed to build the full scale cavity and a high power rf test stand. The main efforts concerning the definition of the construction strategy were focused on the integration of the stems inside the tank, the cooling system, 


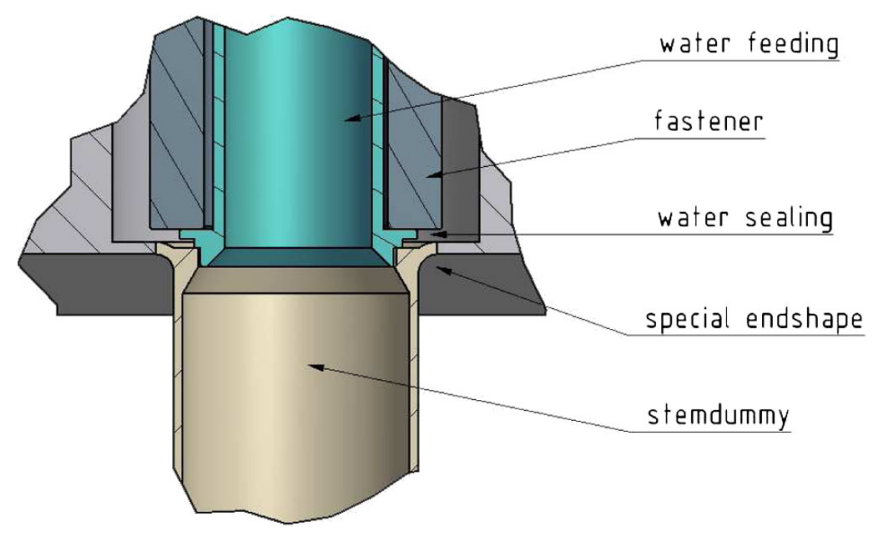

FIG. 45. Schematic view of the connection between the stem and the outer cylinder.

and the modeling of the intertank units for each coupled resonator.

A test model successfully demonstrated the possibility to weld the stem into the outer cylinder on the inner surface. Nevertheless, to reduce the risk of misalignments and rotation of the stems during the welding process, particular care must be taken to limit stress along the stem caused by temperature differences between tank wall and drift tube structure. This led to the design of the contact area which is schematically shown in Fig. 45.

A camera-assisted welding technique was tested at the mechanical workshop of Frankfurt University which demonstrated the possibility to weld the stems with very high

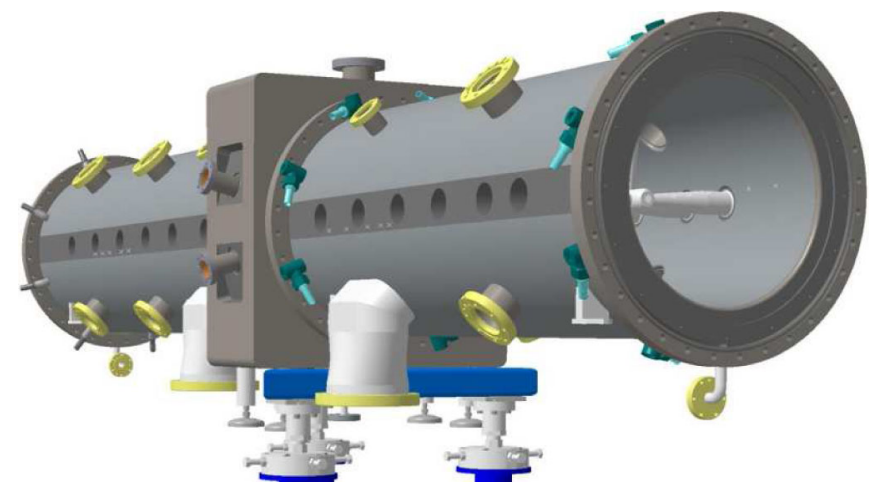

FIG. 46. A model view of the first section of the proton injector.

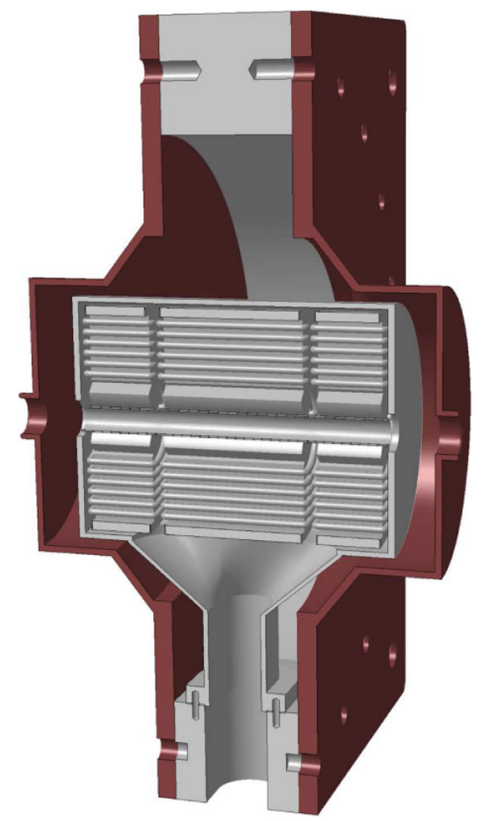

FIG. 48. A 3D view of the intertank section between two $\mathrm{CH}$ cavities including the quadrupole triplet.

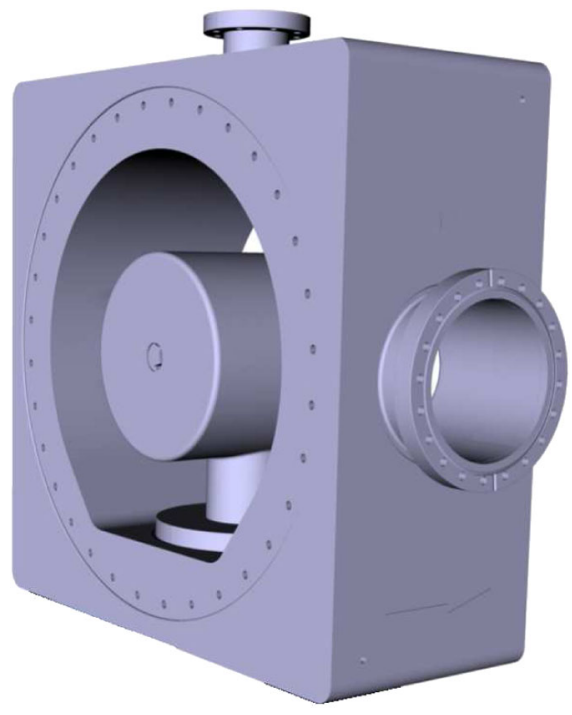

FIG. 49. A 3D view of the coupling section between two coupled $\mathrm{CH}$ cavities.

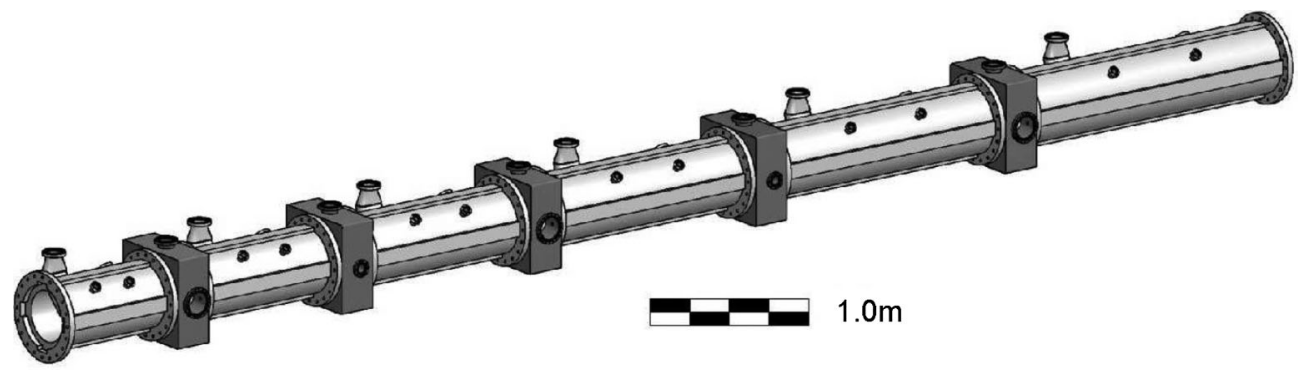

FIG. 47. Mechanical design of the second cavity of the FAIR proton injector. 
precision [55]. Moreover, this technique is fully acceptable for the following galvanic copper plating. With respect to cooling system, the drift tube geometry was modeled to produce the stems by only four single parts. Investigations on the cooling system [56] showed that it is possible to keep the whole cavity at moderate temperature with only eight cooling channels on the tank wall and at a wall thickness of $5 \mathrm{~mm}$ along the stems.

Another critical point concerning the mechanical layout is represented by the intertank unit which has to connect two neighbored cavities. The general mechanical layout of the FAIR proton injector is based on a very compact solution where the entire linac is grouped in two sections, each representing mechanically a stiff unit.

Figure 46 shows the $9 \mathrm{~m}$ long low energy section, which consists of three coupled cavities. The coupling cells and the intertank units with their quadratic outer shape will be directly connected with the alignment and support structure as shown in Fig. 47.

Limited longitudinal motion on rail will be provided for maintenance of individual tank components, a solution already in use at the UNILAC.

For vacuum reasons, the intertank lenses will be housed in thin walled drift tubes, as shown in Fig. 48. Inside each intertank unit a beam position monitor will be integrated to control and correct the bunch center position along the whole linac. A similar general concept is applied to the coupling cell (see Fig. 49). In this case the rectangular flange includes the water cooled lens and the ports for the rf coupler, a tuning plunger, and a pump.

The construction of the second coupled resonator of the proton injector was started in November 2010. The first klystron was already delivered to GSI and the integration of the power supply is expected to be completed within 2011. Within 2012 the cavity will be tested under full power conditions at the new rf test bench at GSI.

\section{SUMMARY AND OUTLOOK}

The rf properties of the CH-DTL have been systematically investigated at the University of Frankfurt together with GSI showing promising results. In particular, the effective shunt impedance is particularly high in the low and medium velocity range which makes that kind of cavity a valid alternative to Alvarez DTL's. A first model demonstrated the feasibility of the crossbar design in terms of mechanical stability, cooling, and copper plating. The experimental results were in good agreement with simulations. A novel scheme based on a large half drift tube in combination with an inclined stem has been developed for an efficient tuning of the end cell.

An innovative coupling concept, where two $\mathrm{CH}$ cavities are coupled through an intertank section resonating in the $E_{010}$ mode, was developed and investigated as well. The validity of this innovative scheme has been verified with a scaled model of the second cavity of the FAIR proton linac. The coupled $\mathrm{CH}$ concept is particularly profitable at low velocity, especially if large power rf amplifiers are available. At higher energies where the rf defocusing effect is less of a concern, the KONUS allows the use of lens-free $\mathrm{CH}$ cavities with reasonable power requests per resonator.

Based on those promising results, GSI decided to base all current linac activities on $\mathrm{CH}$ cavities including the superconducting $\mathrm{cw}$ linac and an optional UNILAC energy upgrade. In particular, the FAIR proton injector will be the first complete linac entirely based on CH-DTL. The prototype of the second cavity of the FAIR proton linac is under construction. This first completed coupled $\mathrm{CH}$ cavity will be tested under full power with a 3.0 MW klystron at the new rf test bench of GSI. Finally, the R\&D activity performed on the $\mathrm{CH}$ cavity could lead that cavity to become a valid and proven alternative to classical DTL structures at low and intermediate beam velocities. If high power amplifiers are available, use of the coupled $\mathrm{CH}$ cavity concept is profitable for energies up to about $40 \mathrm{AMeV}$.

\section{ACKNOWLEDGMENTS}

The authors would like to thank the members of the GSI Galvanic workshop and of the mechanical IAP workshop for the excellent work concerning the copper plating operation of the test cavity, and for the construction of the $\mathrm{CH}$ models, respectively. We also thank M. Bush from IAP, University of Frankfurt, for performing all the rf simulations required for the analytical model of the CH-DTL. The authors deeply express their gratitude to the late Sergey Minaev from ITEP, Moscow, for his inspiring discussions, friendship, and helpful scientific collaboration during the past years. This work has been supported by the Federal German Ministry for Education and Research BMBF06FY9089I and by HIC for FAIR within the LOEWE funding program of the State of Hesse.

[1] H. V. Smith, in Proceedings of the Second Workshop on Utilization and Reliability of High Power Proton Accelerators, Aix-en-Provence, France (OECD Publishing, France, 1999), pp. 79-86.

[2] H. V. Smith, in Proceedings of the Second ICFA Advanced Accelerator Workshop on the Physics of High-Brightness Beams, Los Angeles, CA, edited by James Rosenzweig and Luca Serafini (2000), pp. 466-478.

[3] L.M. Young et al., in Proceedings of the Second ICFA Advanced Accelerator Workshop on the Physics of High-Brightness Beams, Los Angeles, CA (Ref. [2]), pp. 488-502.

[4] Technical Report, SNS Parameter List, 2005.

[5] P. Willendrup et al., in Proceedings of the American Conference on Neutron Scattering, Santa Fe, NM (2008), pp. 11-15.

[6] R.C. York, in Proceedings of the 23rd Particle Accelerator Conference, Vancouver, Canada, 2009 (IEEE, Piscataway, NJ, 2009), pp. 70-74. 
[7] O. Meusel et al., in Proceedings of the 2006 Linear Accelerator Conference, Knoxville, TN, USA, edited by S. Henderson (ORNL, Oak Ridge, 2006), pp. 159-161.

[8] J.L. Biarotte, in Proceedings of the 2004 Linear Accelerator Conference, Lübeck, Germany, edited by D. Trines (DESY, Hamburg, 2004), pp. 275-279.

[9] Technical Report, IFMIF International Team, IFMIF Comprehensive Design Report, International Energy Agency, 2004.

[10] Y. Yamazaki et al., KEK Technical Report No. 2002-13.

[11] L. Arnaudon et al., Linac4 Technical Design Report No. CERN.AB.2006.084 ABP/RF, edited by F. Gerigk and M. Vretenar, 2006.

[12] M. Baylac et al., in Proceedings of the HB 2006 Conference, Tsukuba, Japan, edited by Y.H. Chin, H. Yoshikawa, and M. Ikegami (KEK, Tsukuba, 2006), pp. 137-139.

[13] U. Ratzinger, in Proceedings of the 1991 Particle Accelerator Conference, San Francisco, CA, 1991 (IEEE, New York, 1991), pp. 567-571.

[14] U. Ratzinger, Nucl. Instrum. Methods Phys. Res., Sect. A 464, 636 (2001).

[15] U. Ratzinger, in Proceedings of the European Particle Accelerator Conference, Vienna, 2000 (EPS, Geneva, 2000), pp. 98-102.

[16] W. Barth et al., in Proceedings of the 23rd Particle Accelerator Conference, Vancouver, Canada, 2009 (Ref. [6]), pp. 4905-4907.

[17] S. Minaev et al., Phys. Rev. ST Accel. Beams 12, 120101 (2009).

[18] J. P. Carneiro et al., Nucl. Instrum. Methods Phys. Res., Sect. A 606, 271 (2009).

[19] S.S. Kurennoy et al., in Proceedings of the 2011 International Particle Accelerator Conference, San Sebastian, Spain, 2011, edited by O. Brüning, pp. 26552657.

[20] F. Naito et al., in Proceedings of the 20th Particle Accelerator Conference, Portland, OR, 2003 (IEEE, New York, 2003), pp. 2835-2837.

[21] J. Stowell, in Proceedings of the 2004 Linear Accelerator Conference, Lübeck, Germany, pp. 108-112.

[22] M. Vetrenar et al., CARE Report No., p. 3.

[23] H. Podlech et al., Phys. Rev. ST Accel. Beams, 10, 080101 (2007).

[24] F. Naito et al., in Proceedings of the XX International Linac Conference, Monterey, CA, edited by A. W. Chao (SLAC, Stanford, 2000), pp. 563-565.

[25] J. Broereet et al., in Proceedings of the 19th International Linear Accelerators Conference, Chicago, Illinois, 1998 (NTIS, Springfield, VA, 1998), pp. 57-59.

[26] U. Ratzinger and R. Tiede, Nucl. Instrum. Methods Phys. Res., Sect. A 415, 229 (1998).

[27] Y. Lu et al., in Proceedings of the 2004 Linear Accelerator Conference, Lübeck, Germany, pp. 57-59.

[28] U. Ratzinger, in Proceedings of the 1991 Particle Accelerator Conference, San Francisco, CA, 1991 (Ref. [13]), pp. 567-571.

[29] S. Richter et al., in Proceedings of the 8th European Particle Accelerator Conference, Paris, 2002 (EPS-IGA and CERN, Geneva, 2002), pp. 1181-1183.
[30] E. Nolte et al., in Nucl. Instrum. Methods 158, 311 (1979).

[31] U. Amaldi et al., Nucl. Phys. B172, 277 (2007).

[32] SNS Parameter List, SNS Report No. 100000000-PL0001R13, 2005, pp. 8-9.

[33] T. Kato et al., in Proceedings of the 1998 Asian Particle Accelerator Conference, Tsukuba, Japan, edited by Y.H. Chin, M. Kihara, H. Kobayashi, N. Akasaka, K. Nigorikawa, and M. Tobiyama (KEK, Tsukuba, Japan, 1998).

[34] S. Ramberger et al., CARE-Report-08-054-HIPPI, 2008.

[35] U. Ratzinger, Habilitationsschrift, Universität Frankfurt, Germany (1999).

[36] H. Podlech, Habilitationsschrift, Universität Frankfurt, Germany (2008).

[37] G. Clemente, Ph.D. dissertation, Universität Frankfurt, 2007.

[38] M. J. Browman et al., in Proceedings of the 1998 Linear Accelerator Conference, Newport, VA, USA (NTIS, Newport News, VA, 1989), pp. 64-66.

[39] G. Clemente et al., in Proceedings of the 21st Particle Accelerator Conference, Knoxville, 2005 (IEEE, Piscataway, NJ, 2005), pp. 883-885.

[40] U. Ratzinger et al., in Proceedings of the 2006 Linear Accelerator Conference, Knoxville, TN, USA, pp. 526-530.

[41] T.P. Wangler, in RF Linear Accelerator (Wiley-VCH, Weinheim, 2008), 2nd ed., pp. 98-100.

[42] I. Wilson and H. Henke, CERN Internal Report No. CERN 89-09, 1989, pp. 16-20.

[43] U. Ratzinger et al., Laser Part. Beams 21, 627 (2003).

[44] W. Henning, FAIR Technical Report, GSI, Darmstadt (2001).

[45] W. Barth et al., in Proceedings of the 2004 Linear Accelerator Conference, Lübeck, Germany, pp. 246-250.

[46] L. Groening et al., Proton Linac Technical Design Report, GSI, Germany (2009).

[47] C. Zhang and A. Schempp, Nucl. Instrum. Methods Phys. Res., Sect. A 609, 95 (2009).

[48] R. Douperrier et al., in Proceedings of the XX International Linac Conference, Monterey, CA (Ref. [24]), pp. 839-841.

[49] Manual of the LANL RFQ Design Codes, Report No. LAUR-96-1836, LANL, revised 3, 2005.

[50] G. Clemente et al., in Proceedings of the 11th European Particle Accelerator Conference, Genoa, 2008 (EPS-AG, Genoa, Italy, 2008), pp. 2701-2703.

[51] G. Clemente et al., in Proceedings of the HB 2006 Conference, Nashville, TN, USA, edited by S. Henderson and M.A. Hensley (National Laboratory Spallation Neutron Source, Oak Ridge, 2008), pp. 883-885.

[52] R. Tiede, Ph.D. dissertation, Universität Frankfurt, 2007.

[53] L. Groening et al., Phys. Rev. ST Accel. Beams, 11, 094201 (2008).

[54] M. Busch et al., in Proceedings of the 2009 SRF, Berlin, Germany, edited by M. Abo-Bakr, B.C. Kuske, A. Liebezeit, S. Voronenko, and B. Schaa (HelmholtzZentrum, Berlin, 2009), pp. 575-577.

[55] R. Brodhage et al., in Proceedings of the 2010 International Particle Accelerator Conference, Kyoto, Japan (ICR, Kyoto, 2010), pp. 750-752.

[56] R. Brodhage et al., in Proceedings of the 23rd Particle Accelerator Conference, Vancouver, Canada, 2009 (Ref. [6]). 